\title{
Orbital evolution of the Carina dwarf galaxy and self-consistent determination of star formation history ${ }^{\star}$
}

\author{
S. Pasetto ${ }^{1,2}$, E. K. Grebel ${ }^{1}$, P. Berczik ${ }^{1,4,5}$, C. Chiosi $^{3}$, and R. Spurzem ${ }^{1,4}$ \\ 1 Astronomisches Rechen-Institut, Zentrum für Astronomie der Universität Heidelberg, Germany \\ e-mail: spasetto@ari.uni-heidelberg.de \\ 2 Max-Planck-Institut für Astronomie, Heidelberg, Germany \\ 3 Department of Astronomy, University of Padova, Italy \\ ${ }^{4}$ National Astronomical Observatories of China (NAOC), Chinese Academy of Sciences (CAS), Datun Lu 20A, Chaoyang District, \\ Beijing 100012, PR China \\ 5 Main Astronomical Observatory (MAO), National Academy of Sciences of Ukraine (NASU), Akademika Zabolotnoho 27, \\ 03680 Kyiv, Ukraine
}

Received 7 October 2009 / Accepted 8 September 2010

\section{ABSTRACT}

\begin{abstract}
We present a new study of the evolution of the Carina dwarf galaxy that includes a simultaneous derivation of its orbit and star formation history. The structure of the galaxy is constrained through orbital parameters derived from the observed distance, proper motions, radial velocity, and star formation history. The different orbits admitted by the large proper motion errors are investigated in relation to the tidal force exerted by an external potential representing the Milky Way. Our analysis is performed with the aid of fully consistent $\mathrm{N}$-body simulations that are able to follow the dynamics and the stellar evolution of the dwarf system in order to determine the star formation history of Carina self-consistently. We also find a star formation history characterized by several bursts, partially matching the observational expectation. We find also compatible results between dynamical projected quantities and the observational constraints. The possibility of a past interaction between Carina and the Magellanic Clouds is also separately considered and deemed unlikely.
\end{abstract}

Key words. galaxies: dwarf - Local Group - galaxies: kinematics and dynamics - celestial mechanics - galaxies: evolution

\section{Introduction}

It has been extensively reviewed by many authors (e.g., Grebel 1999; van den Bergh 1999; Mateo 1998) that dwarf spheroidal galaxies (dSphs) are the most common type of galaxies in the Local Group (LG). Their origin and evolution are focal points because of the key role that they play in the context of the hierarchical growth of structures in the Universe and because of the natural interest that these peculiar systems engender as examples of dark-matter dominated systems. In the context of dynamical research on interacting systems, dSphs are also crucial as stellar systems found in the central regions of galaxy clusters and groups, thus becoming potential subjects of strong gravitational interactions. This morphology-position relation is also observed in our LG (e.g., Grebel 1999).

Tidal interactions may leave a variety of structural signatures (see, for instance, Read et al. 2006; Muñoz et al. 2008; Peñarrubia et al. 2008, 2009). In the LG, Sagittarius is the bestknown example of a currently disrupting dwarf galaxy but, based on photometric studies tidal interactions, have also been claimed for other dSphs such as Ursa Minor, Sculptor, and Carina (e.g., Eskridge 1988a,b; Irwin \& Hatzidimitriou 1995; Kuhn et al. 1996; Smith et al. 1997; Majewski et al. 2005, 2000; MartínezDelgado et al. 2001; Palma et al. 2003; Muñoz et al. 2005; Walcher et al. 2003; Westfall et al. 2006; Wilkinson et al. 2004; Sohn et al. 2007; Muñoz et al. 2006; Majewski et al. 2003; Strigari et al. 2008). Draco, on the other hand, appears to be

\footnotetext{
* Appendices are only available in electronic form at http://www. aanda.org
}

an example of an undisturbed dSph despite its proximity to the Milky Way (Odenkirchen et al. 2001; Klessen et al. 2003). Tidal interactions and their effects have also been extensively explored in $N$-body simulations (e.g., Johnston et al. 1999; Mayer et al. 2002; Pasetto et al. 2003). In this context, a general bimodal density profile can be used to describe the systems with an inner stellar population and a shallowly decreasing density profile in the outer regions as a consequence of the tidal interaction. While the external density profile is generally attributed to the tidal interactions, it is unclear whether the inner regions of dSphs are strongly influenced by tides, or whether a high $M / L$ ratio can dampen the tidal gravitational shocks (see Burkert 1997; Kroupa 1997; Gómez-Flechoso et al. 1999; Klessen \& Zhao 2002; Gómez-Flechoso \& Martínez-Delgado 2003; Kleyna et al. 2002; Walker et al. 2006a,b; Gilmore et al. 2007) ${ }^{1}$. The radial velocity dispersion profile is a powerful tool for investigating multi-component self-gravitating systems, since it is sensitive to the dark matter distribution and accessible to observations (e.g., Pasetto et al. 2010). Recently, radial velocity data have become available to track the line-of-sight velocity dispersion as a function of radius for many LG dwarf galaxies (e.g., Pasetto et al. 2010; Tolstoy et al. 2004; Westfall et al. 2006; Wilkinson et al. 2004; Muñoz et al. 2005; Walker et al. 2006a,b, 2009; Sohn et al. 2007; Koch et al. 2007c,b; Muñoz et al. 2006), and these data

\footnotetext{
1 A slightly different approach can be found in Piatek \& Pryor (1995) and Oh et al. (1995) where the authors span, in a specialized context, a wide range of eccentricities for exceedingly low-mass dwarf galaxies without dark matter (but see also Klessen \& Zhao 2002, Klessen et al. 2003).
} 
permit more detailed modelling of the dwarf galaxies kinematic status (e.g., Kroupa 1997; Kleyna et al. 1999; Kazantzidis et al. 2004; Read et al. 2006; Peñarrubia et al. 2008, 2009; Pasetto et al. 2010). For the same approach to multi-component dSphs in the cosmological $\Lambda$ CMD contest see, Stoehr et al. (2002) and Hayashi et al. (2003) or, in a purely dynamical collisonless regime see Ciotti \& Morganti (2009), where the primordial stellar population is embedded in an extended dark matter halo without considering any gas process.

In our current study, we concentrate on the Carina dSph galaxy, for which an extensive body of observational data is available. We explore the orbital evolution of this dwarf. The paper is divided in two main sections: Sect. 2 reviews the observations on Carina and presents a preliminary orbit investigation in a point-mass approximation, while Sect. 3 presents the $N$-body simulations and comparisons with the observations. Conclusions are presented in Sect. 4.

\section{The family of orbits for the Carina dwarf galaxy}

\subsection{Initial condition vs. final condition}

In studying the orbits of the Carina dwarf galaxy, aspects of its actual dynamical and kinematic condition, the history of its stellar populations and chemical enrichment, must be taken into account. The incompleteness of available data, their uncertainty or errors, and the limits of our analysis tools increase the difficulties to overcome in order to produce a realistic model for Carina's orbit. Here, the complete amount of available data for this galaxy is too great to be reviewed fully, thus only the more salient characteristics are reported.

\subsection{Observations}

\subsubsection{Proper motions, distance, and radial velocity}

The present observational data on the Carina dSph make it necessary for us to discuss families of orbits instead of a true single orbit. We devote our main effort to reduce the number of plausible orbits using all the available constraints known to date. We started with choosing the proper motions in Table 1 of Metz et al. (2008) based on advanced charge transfer inefficiency (CTI) correction for the Space Telescope Imaging Spectrograph (STIS): $\left(\mu_{\alpha} \cos \delta, \mu_{\delta}\right)=(+22 \pm 13,+24 \pm 11) \mathrm{mas} \mathrm{yr}^{-1}$. We then proceeded to reduce them to an inertial galactocentric reference system $S_{0}$, updating Johnson \& Soderblom (1987) to J2000 as already done by e.g., Pasetto et al. (2003). We extensively describe the orientation of the velocity space directions for this inertial reference system centred on the MW, $S_{0}$, in Appendix A. The resulting velocity vector for Carina is $\boldsymbol{v}_{\mathrm{Car}}=\{+113 \pm 52,-14 \pm 25,+44 \pm 56\} \mathrm{km} \mathrm{s}^{-1}$. Proper motions for Carina have also been recently derived by Walker et al. (2008) with error bars compatible with the values we adopted here.

The first determination of the distance of Carina comes from the RRLyrae-based distance modulus estimation by Saha et al. (1986), and more ones are from Smecker-Hane et al. (1994), Hurley-Keller et al. (1998), Mighell (1997), and McNamara (1995).

Measurements of the radial velocity have already been made in an older work on carbon stars by Cook et al. (1983), where the authors deduced a radial velocity dispersion of $6 \mathrm{~km} \mathrm{~s}^{-1}$. The actual value we assumed for the radial velocity is $v_{r}=$ $224( \pm 3) \mathrm{km} \mathrm{s}^{-1}$ (Mateo et al. 1998; Mould et al. 1990).
Methodology for the dwarf galaxy simulation: We simulated a dwarf galaxy composed of three components: a dark matter component that dominates the total mass, a gas component and a stellar component. We assume that the preexisting old stellar population has settled after an initial violent-relaxation phase of the dark halo, which created the potential well within which the baryonic component collapsed. The corresponding burst of star formation created the oldest stellar population that is observable through analysis of the observed colour magnitude diagrams (CMD) (see next section). We assumed a time scale of a few Gyr for the realization of this initial 3-component dwarf galaxy model and its subsequent settling down in an orbit around the Milky Way (MW) with an initial orbital energy $E_{\text {orb }}^{\text {ini }}$. We started our simulations after this initial evolution has already taken place, i.e., we consider a dwarf galaxy in which the old population has already formed and that is already in orbit around the MW. We next considered the subsequent orbital evolution covering the last $\cong 9$ Gyr. We thus assumed that the observed proper motions are indirectly related to this initial orbital energy $E_{\text {orb }}^{\text {ini }}$. There is no reason to investigate the orbit prior to this initial time, because the system has still not settled down into thermodynamic/virial equilibrium, and the concept of a barycentre for a complex of blobs of gas and stars is still meaningless. Similarly, by experiencing a collisionless violent relaxation process, any old preexisting stellar population loses memory of its initial phase-space distribution that, as a consequence, is completely unrelated to the currently observable kinematic properties of Carina (see following sections for a review of other possible scenarios of formation for Carina).

\subsubsection{Milky Way model}

In our simulation we included an external galaxy model resembling that of the Milky Way. Nevertheless, considering the large Galactocentric distance of the dwarf galaxy we are going to analyse and the large uncertainties in the MW models, we model the MW with a few simplified assumptions:

1. Milky Way potential. The MW is simplified as a vector field across which we first orbit a point-mass model of the Carina dwarf galaxy (in Sect. 2.3). Then, once we have reduced the plausible orbits suitable to our aims, we orbit a full self-consistent $N$-body model of the Carina dwarf galaxy considering gravity, star formation, and feedback processes of this "live"-satellite. We tested different potentials representing the MW in order to obtain results independent from our parametrization. Nevertheless, we want to stress that the large errors involved in the observational proper motion determination in general do not permit us to easily constrain the MW potential from the orbit determination of its satellites. The fully analytical parametrization is based on a logarithmic halo

$$
\Phi_{\mathrm{h}}(R, z, t)=k_{1}^{2} \log \left(R^{2}+k_{2}^{2}+\frac{z^{2}}{k_{3}^{2}}\right) .
$$

For the disk we can choose a potential formulation as (after Miyamoto \& Nagai 1975)

$$
\Phi_{\mathrm{d}}(R, z, t)=-\frac{G M_{\mathrm{d}}}{\sqrt{R^{2}+\left(k_{4}+\sqrt{z^{2}+k_{5}^{2}}\right)}},
$$

and for the bulge

$\Phi_{b}(R, z, t)=-\frac{G M_{\mathrm{b}}}{\sqrt{R^{2}+z^{2}}+k_{6}}$, 
Table 1. Parameters adopted as starting and final values for analytical MW potential of Eqs. (1)-(3).

\begin{tabular}{lcccccccc}
\hline \hline$t_{\text {ini }}$ & $\begin{array}{c}M_{\mathrm{b}} \\
{\left[M_{\odot}\right]}\end{array}$ & $\begin{array}{c}M_{\mathrm{d}} \\
{\left[M_{\odot}\right]}\end{array}$ & $\begin{array}{c}k_{1} \\
{\left[\mathrm{~km} \mathrm{~s}^{-1}\right]}\end{array}$ & $\begin{array}{c}k_{2} \\
{[\mathrm{kpc}]}\end{array}$ & $k_{3}$ & $\begin{array}{c}k_{4} \\
{[\mathrm{kpc}]}\end{array}$ & $\begin{array}{c}k_{5} \\
{[\mathrm{kpc}]}\end{array}$ & $\begin{array}{c}k_{6} \\
{[\mathrm{kpc}]}\end{array}$ \\
\hline $\begin{array}{l}\text { Bulge } \\
\text { Disk }\end{array}$ & $3.4 \times 10^{10}$ & & & & & & & 0.7 \\
Halo & & $0.9 \times 10^{11}$ & & & & 6.5 & 0.26 & \\
\hline$t_{\text {end }}$ & & & 120.8 & 10 & 1 & & & \\
\hline $\begin{array}{l}\text { Bulge } \\
\text { Disk }\end{array}$ & $3.4 \times 10^{10}$ & & & & & & & 0.7 \\
Halo & & $1.0 \times 10^{11}$ & & & & 6.5 & 0.26 & \\
\hline
\end{tabular}

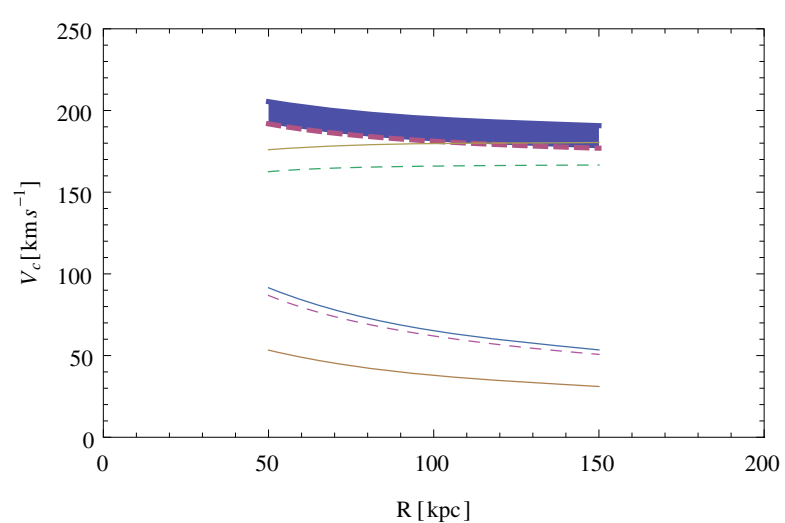

Fig. 1. Rotational curve of the growing halo of the MW potential. The shadow represents the zone of gained mass for accretion in the outer part of the halo.

where the dependence on the position is made explicit by the cylindrical parametrization $(O, R, z)$ of $S_{0}$, while the dependence on the time $t$ is implicit in the parameter $k_{i}=k_{i}(t)$ for $i=1, \ldots, 6$ and on the total masses $M_{\mathrm{d}}=M_{\mathrm{d}}(t), M_{\mathrm{b}}=M_{\mathrm{b}}(t)$ for the disk and the bulge, respectively. Here, $G$ is the gravitational constant $G=6.673 \times 10^{-11} \mathrm{~m}^{3} \mathrm{~kg}^{-1} \mathrm{~s}^{-2}$. Particular attention is paid to the tuning of these parameters in order to match the now known constraints on the MW potential. The adopted parameter are listed in Table 1 .

The evolution of the rotation curve for the radial range of Galacocentric distances of interest for the Carina's orbits is shown in Fig. 1. The upper thick blue line is, for instance compatible with the recent determination by Gnedin et al. (2010) even if a larger margin of error in the rotation curve is actually permitted in relation to the Sun's location (e.g., Reid et al. 2009; Bovy et al. 2009; Sirko et al. 2004b).

We stress that different studies have found differences for the MW mass inside to relevant zone for our orbit integration. E.g., Kochanek (1996) obtained $M_{\text {tot }}(r<100 \mathrm{kpc})=$ $7.5 \times 10^{11} M_{\odot}$, and Dehnen \& Binney (1998) obtained $M_{\text {tot }}(r<100 \mathrm{kpc})=7 \times 10^{11} M_{\odot}$. In addition, many different approaches can produce mass estimates for the MW; e.g., Zhao (1998) used the velocity distribution of the MW satellites and Robin et al. (2003) used the escape velocity from the local standard of rest and Combes (1999) from timing arguments. Similar results were found by Sakamoto et al. (2003) from the kinematics of Galactic satellites, halo globular clusters, and horizontal branch stars. Lin et al. (1995) obtained $M_{\text {tot }}(r<100 \mathrm{kpc})=5 \times 10^{11} M_{\odot}$ from the dynamics of the Magellanic Clouds, Vallenari et al. (2004) found $M_{\mathrm{tot}}(r<100 \mathrm{kpc})=8.8 \times 10^{11} M_{\odot}$ from proper motion modelling of stars in two deep fields toward the North
Galactic Pole. Mass estimates based on the moments of the Jeans equation are often sensitive to the anisotropy profile of the MW (e.g., Brown et al. 2010) leading to unsecured results, e.g. compare Battaglia et al. (2005) and Dehnen et al. (2006), or Watkins et al. (2010) who quote a value for $M_{\mathrm{tot}}(r<300 \mathrm{kpc}) \approx 1.4 \times 10^{12} M_{\odot}$, and recently Xue et al. (2008) have found $M_{\text {tot }}(r<60 \mathrm{kpc}) \approx 4.0 \times 10^{11} M_{\odot}$.

2. Milky Way accretion rate. We expect that, in the last $9 \mathrm{Gyr}$ of evolution, not only did the dwarf galaxy change its physical properties, but also the mass of the MW grew from the continuous inflow of matter. For simplicity we assume that

- the MW has not experienced any major merger in the last 9 Gyr, and

- star formation processes are of minimal importance in the growth of MW.

These simplifications are realistic for our time range of $\cong 9$ Gyr (e.g., Guo \& White 2008). What is more interesting to us is the evolution of the density distribution in the outer part of the halo (see Helmi (2008) for a general review), because the outer halo is where the orbit of a dwarf galaxy is expected to pass through. We represent the external mass distribution of the MW with an external potential that depend on time.

All these determinations lead to a wide range of values within which we want to choose our parameters when a growth factor is added in a time-dependent potential for the halo component. For the MW we assume that the growth rate is mainly due to the minor mergers that are expected to be dominant for redshift $z<1$ and for galaxies as massive as MW (Guo \& White (2008)). Taking the uncertainties into account in the final target amount of dark matter and the mass-to-light ratio of the MW as in Flynn et al. (2006), a simple linear growth factor of $10 \div 25 \%$ is tested for the MW in the last 9 Gyr of evolution $(z<1.46$ in redshift) (e.g., Combes 1999; Guo \& White 2008). Higher values for this parameter can be found in the literature, although the relevance of the MW mass growing factor is limited by the large uncertainty in the total mass of the MW $\left(M_{\mathrm{tot}}(r<100) \mathrm{kpc}\right)$. This uncertainty has an unavoidably bad influence on our ability to determine the orbits of all the dwarf galaxies in the MW outskirts. We also test and take a gradual flattening of the inner halo into account, although no data exist on Carina dwarf galaxy that are sensitive to this parameter; e.g., it can be proved, as in Pasetto et al. (2003) that the presence of the bulge is not relevant to integration of the orbit of Carina (similar to Sculptor in Pasetto et al. (2003) and the following sections). The only observational data, (e.g., from the SDSS Sirko et al. 2004a; Jurić et al. 2008), refer to the first $25 \mathrm{kpc}$ of Galactocentric distance and are extremely unlikely in the orbit of Carina (see Figs. 2 and 4 in the following section). 
3. Milky Way triaxiality. The MW potential and the possibility if its triaxiality are investigated in different papers such as Helmi (2004), Martínez-Delgado et al. (2007). Unfortunately, the approach in these studies is based on the possibility of tracking the orbits with the help of tidal streams (e.g. Cole et al. 2008; Eyre \& Binney 2009; Koposov et al. 2010) and thus is limited to a few objects, mainly globular clusters or to the Sagittarius dwarf galaxy. Our target, the Carina dwarf galaxy, does not show any long tidal streams that would permit us to constrain the MW potential triaxiality. Despite the simplicity of implementing a tiaxialhalo MW, we do not introduce here such a potential since its influence cannot be directly verified within the errors of our present work. Moreover, a triaxial halo presents challenges the stability of the disks as shown in Kazantzidis et al. (2010), the origin and evolution of the dark matter axis ratios $c / a$ and $c / b$ are still unclear, and the relative orientations of the three axes are still a matter of debate. Nevertheless, we stress that the role of triaxiality is invoked as a fundamental tool in investigating the orbits of the MW satellites close to the galactic centre (e.g., Law et al. 2009).

\subsubsection{Star formation history}

Many authors have investigated the star formation history (SFH) of the Carina dSph since its discovery by Cannon et al. (1977) and the pioneering work of Mould \& Aaronson (1983). These studies demonstrate the presence of multiple populations with different ages (Azzopardi et al. 1985, 1986; Smecker-Hane et al. 1994; Mould et al. 1990; Mighell 1997; Hurley-Keller et al. 1998; Mighell 1990a). Monelli et al. (2003a) published a deep CMD for the central zones of Carina, and Rizzi et al. (2003) derive the SFH from a more spatially extended sample based on analysis of RGB stars. We can then assume that Carina experienced an initial burst of star formation that formed the oldest stellar population a second strong burst around $\sim 6 \mathrm{Gyr}$ ago, and a more recent event around $\sim 2$ Gyr ago. These episodes of star formation are clearly visible in Carina's CMD, and the existence of a fourth, even more recent episode has been proposed. What makes Carina special is that these episodes are recognizable as distinct events with distinct main sequence turn-offs.

\subsubsection{Chemical evolution}

Constraints on the Carina metallicity distribution have only recently come from the spectroscopic studies or chemical evolution models (e.g., Koch et al. 2006, 2007a, 2008; Lanfranchi et al. 2006; Abia et al. 2008). They generally show a large spread in the metallicity distribution. We will consider the chemical evolution in more detail in a separate work (Pasetto et al., in preparation).

\subsection{Point mass integrations}

Since our work is based on two full $N$-body integrators (e.g., Berczik 1999; Carraro et al. 1998), which can track many different physical processes simultaneously and produce results that are compatible with observational constraints, one might reasonably question the utility and validity of point-mass approximations. We contend that there are multiple advantages in using of

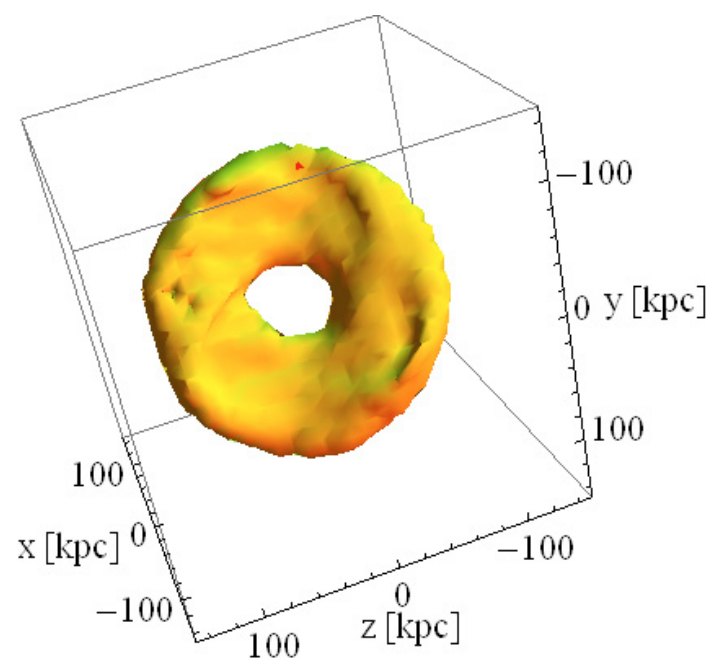

Fig. 2. Error propagation for the point-mass approximation in the orbit of the Carina dwarf galaxy for $10^{5}$ orbits.

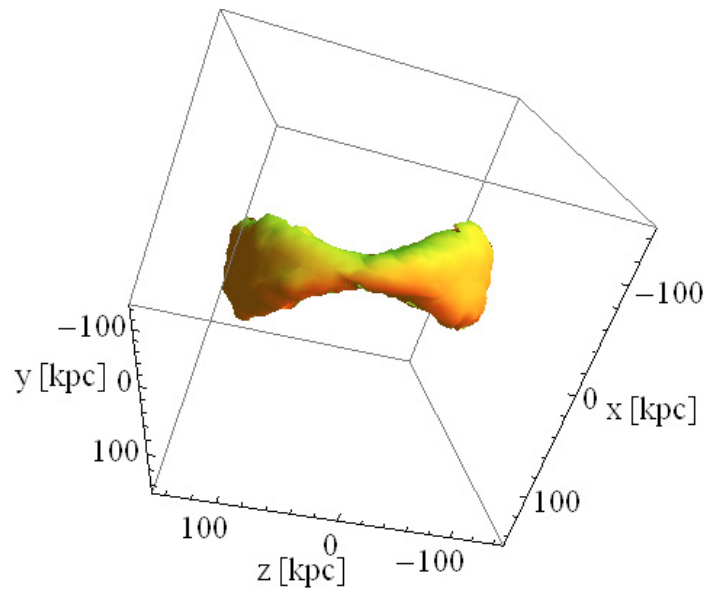

Fig. 3. Same as Fig. 2 but with an edge-on view showing the box-type shape of the orbit and the permitted regions.

the point-mass approximation as a preliminary study for orbit determination:

1. The principal effect that can influence orbit determination is the mass loss that the dwarf suffers during its pericentric passages. This mass change cannot easily be parametrized because it depends on the intensity of tidal effects, as well as on the initial internal structure of the dwarf. Thus we use phase-space coordinates and velocities obtained from pointmass calculations to get the initial values of the Carina orbit for the full $N$-body simulations only for the first $\cong 9 \mathrm{Gyr}$ of backward evolution, i.e., before the time of the first pericentric passage $t_{\mathrm{p} 1}$. These initial guess values for the phasespace location of the barycentre of the $N$-body system span the full range of positions in configuration space from the approximate location of the apocentre (where nowadays we see Carina and we make an approximate match with the observations) to first pericenter (at the time $t=t_{\mathrm{p} 1}$ ) and lead to different star formation histories even if they correspond perfectly to the same initial orbital energy. As an example of the orbit indetermination resulting from the large error bars on the proper motion, we plot in Figs. 2-4 the orbit integrations, backward in the time $t$, for point-mass particles where initial values have been randomly sampled with uniform distribution inside the error bars for the velocities. 


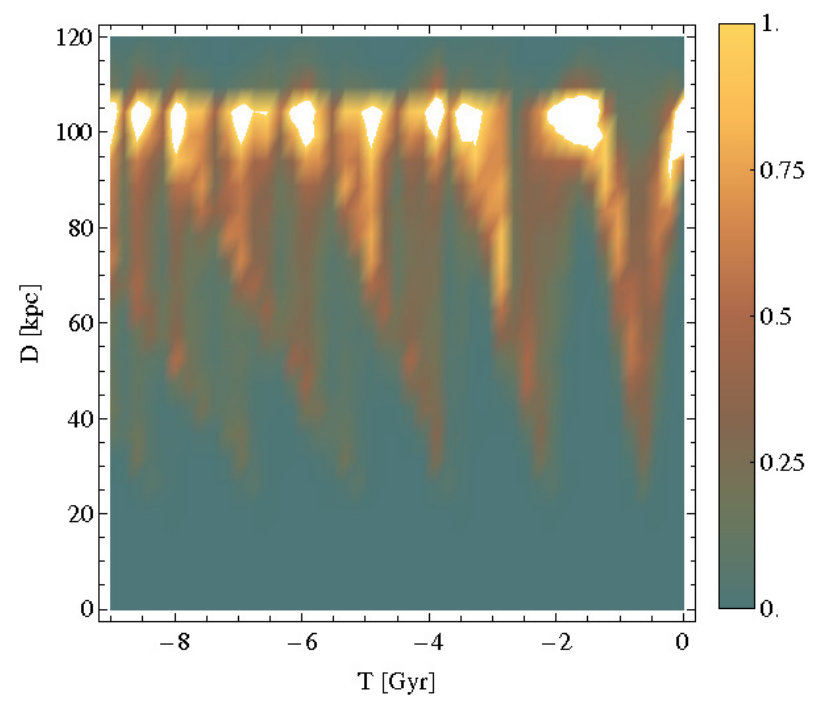

Fig. 4. The radial distance of the dwarf galaxy barycentre with respect to the inertial reference system centred on the MW. The probability of the location of the dwarf over the look-back time has been computed. Larger white zones (i.e., high probability) are closer to the present time where the indetermination has a minor impact.

2. The symmetries of the orbits remain evident. The expected appearance of the orbit of Carina from the point-mass integration is roughly a rosette-like orbit with several pericentre passages. This observation permits us to make some simplifying assumptions. The old stellar disk population of the MW is at least as old as 10 Gyr (e.g., Freeman \& Bland-Hawthorn 2002), thus we can assume that the gravitational potential of the disk and the bulge of MW has not changed its gravitational influence on the orbit of Carina. This allows to us to limit the growth rate in the MW mass only to the halo component, keeping the amount of mass fixed in the disk and the bulge. A growing disk was nevertheless tested in our simulation as explained in Table 1.

3. The rosette orbit symmetry reflects a time symmetry that permits us to backward-integrate the $N$-body gravitational system by simply changing the sign of the velocities. Nevertheless, the present structure to which we apply the orbital phase space and the external gravitational influence of the MW describes a possible initial dynamical structure of the system as it was after the oldest stellar population had formed. It is often not considered in dynamical studies that the stellar evolution history does not have the time symmetry that is assumed in the purely dynamical approach. Clearly, the gas consumption and the chemical evolution are unidirectional even if the dynamical regime is completely collisionless. Thus the point-mass integration provides us with an initial volume of the phase space values to assign to a full $N$-body simulation model at the starting time, say $t=t_{0}=9$ Gyr ago.

Even though it is clear that the orbital plane determination of full $\mathrm{N}$-body simulation and a point-mass integration in a spherical potential is not supposed to be too different between, we would like to show that this is also the case for the pericentre passages in this type of orbit. This is done in Fig. 5 where we have plotted the results of the point-mass integration for an orbit with a close pericentre passage for the set allowed by Carina's proper motion error bars. The same initial conditions were then evolved in a fully consistent $N$-body simulation. The results were obtained with two different codes: the first by Berczik (1999) in

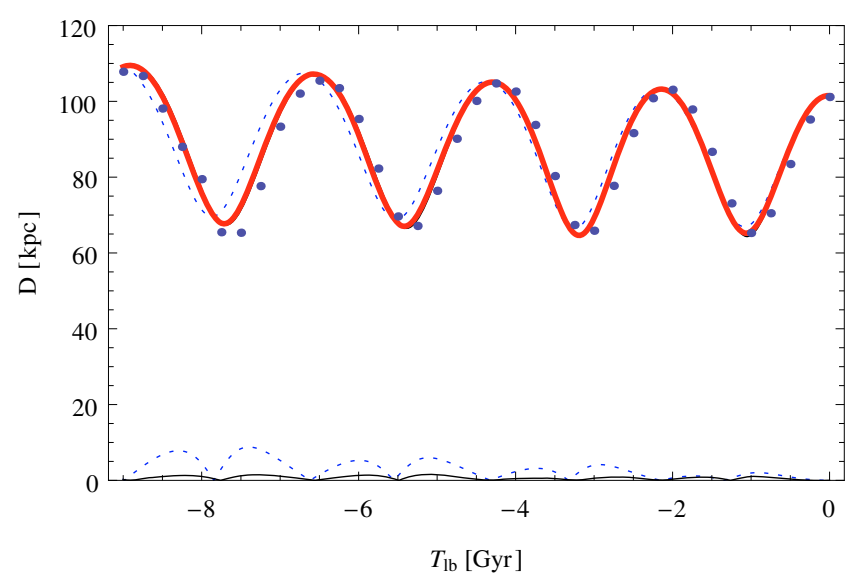

Fig. 5. Radial distance evolution test. Comparing the $N$-body simulation (black dots) and the point-mass integration (solid red line). The dashed line represents the integration without the bulge mentioned in Sect. 2.5. The lines at the bottom represent the difference between the red line and the dashed line, while the solid black line represents the difference between the red line and the orbit computed considering the dynamical friction (see text for details and Sect. 2.5).

a serial machine with special hardware dedicated (see Spurzem et al. 2009) and the second by Carraro et al. (1998) on a parallel machine at the Juelich Super-computer Centre. Both codes yield comparable results across the entire range of initial values.

Why then our approximation work so well? If we consider the two galaxies MW and Carina, we have to treat them as extended bodies. In this case the acceleration of the centre of mass of Carina is given by

$m_{\mathrm{I}}^{\mathrm{Car}} \frac{\mathrm{d}^{2} \boldsymbol{x}}{\mathrm{d} t^{2}}=-\int \rho_{\mathrm{G}}^{\mathrm{Car}}\left(\boldsymbol{x}^{\prime}\right) \nabla_{\boldsymbol{x}^{\prime}} \Phi_{M W}\left(\boldsymbol{x}^{\prime}\right) \mathrm{d}^{3} \boldsymbol{x}$

where the integral is extended to the entire galaxy, and we can no longer assume $\nabla_{x^{\prime}} \Phi_{M W}\left(x^{\prime}\right) \cong$ const. to take it outside the integral sign. This means that extended bodies orbit generally with the same velocities as a point particle if and only if $\nabla_{\boldsymbol{x}^{\prime}} \Phi\left(\boldsymbol{x}^{\prime}\right) \cong$ const., i.e. if $\nabla_{\boldsymbol{x}^{\prime}} \Phi\left(\boldsymbol{x}^{\prime}\right)$ is roughly constant within the body volume of the Carina dwarf galaxy and if it can be extracted off the integral sign to obtain the point mass equation of motion. In general, this approximation cannot be applied to galaxies because they are not bodies that could be considered small enough such as in the case of a globular cluster orbiting around the central zones of the bulge. Nor can they move in a sufficiently homogeneous gravitational field; e.g., they can reach the inner zones of the MW galaxy where the bulge influence become dominant (see Sect. 2.5). Using the consideration the MW potential given in the previous section we made certain to check that the orbital range of Carina in the external potential of the MW satisfies the required approximation in every orbit computation performed with the point-mass approximation and in minimizing the action (see next section). Thus we safely proceed with the approximation $m_{\text {Car }}(t) \cong m_{\text {Car }}=$ const. and the pointmass approximation in the next section where we develop a dedicated method to select a preferential initial condition for the full $N$-body simulations. In the $N$-body simulations explained in the next section mass loss effects will be self-consistently taken into consideration. 


\subsection{Minimizing the action}

After these preliminary considerations it became evident that, although the the errors in the proper motions are quite large, at least the orbital plane can be inferred relatively easily from the orbit analysis. For any chosen value within the range permitted by the error bars of the proper motions, the orbital plane remains roughly determined. The range of orbits allowed by the error bar permits us to deduce that the orbits are more likely box-type. It results not from the orbit determination, which is integrated for too short a time range, but from the consideration of a full sample of integrated orbits.

The true orbit is probably contained in this plane, but we want to proceed by constraining the possible orbits further with an alternative approach. The approach of using a point mass for the orbiting dwarf galaxy can bias the determination of the pericentre and apocentre of the orbit evolution if the dwarf passes very close to the inner zones of the galaxy. Nevertheless, we do not expect it to influence the plane determination of the orbit, since the Carina dwarf galaxy is orbiting mostly in the external regions of a growing MW halo, nor do we expect it to be so large to affect the error estimates of the pericentre position or to invalidate the following considerations. To support these approximations, we remember that, when Carina is more massive (before the first pericentre passage), the MW halo still has its spherical shape, which is gradually converted into an ellipsoidal shape at $t_{\text {end }}$ with $k_{3}$ parameter changing as in Table 1) from 1 to 0.8 . Based on Fig. 4, we can prove that the probability that Carina intersects the disk of the MW is null and the probability of a pericentre lower than $60 \mathrm{kpc}$ is only a few percent!

The generic Lagrangian of the dynamical MW-Carina system can be written down as

$L=\frac{1}{2} m_{\mathrm{Car}}\|\dot{\boldsymbol{x}}(t)\|^{2}-m_{\mathrm{Car}} \Phi_{\mathrm{Gal}}(\boldsymbol{x}, t)$.

The Lagrangian is a function that lives in the tangent bundle of the configuration manifold $\mathbb{Q}$ of Figs. 2 or 3 , so it is defined in $L: T \mathbb{Q} \times \mathbb{R}_{0}^{-} \rightarrow \mathbb{R}_{0}^{-}$(where $\mathbb{R}_{0}^{-}$is the (negative) time line parametrized by $t$ ) and its integral can be easily solved numerically. Thus, we ask ourselves what the orbital plane is within the error bars of the proper motion that minimizes the action. The principle of minimal action will lead us to possible stationary points for the integral of the Lagrangian; hence, we proceed by minimizing the action integral $S \equiv \delta \int_{t_{0}}^{0} L \mathrm{~d} t$, subject to the constraints on the initial value that we deduce from the proper motion and the star formation history. We can, in fact, suppose that the episodes of star formation have been triggered by the tidal influence of the MW potential in the pericentre passages. The proof of this hypothesis will come only in the next section. Although in $T \mathbb{Q}$ the equations of motion are a set of first-order differential equations (i.e., it passes just one solution of the equations of motion through each point $(\boldsymbol{x}, \dot{\boldsymbol{x}})$ of $T \mathbb{Q})$ thus permitting us to easily integrate the action $S$, before performing a minimization of the action we can already restrict the space $T_{x_{\mathrm{Car}}(0)} \mathbb{Q}$, i.e. the fibers (in the geometrical sense) of the Carina configuration position at the initial time $\boldsymbol{x}_{\mathrm{Car}}(0)$.

In terms of Newtonian dynamics, we already gain a more restrictive velocity space than $V^{3} \equiv$ $\left\{\boldsymbol{v}: \boldsymbol{v} \in \mathbb{R}^{3} \wedge \boldsymbol{v}-\sigma_{\boldsymbol{v}} \leqslant \boldsymbol{v} \leqslant \boldsymbol{v}+\sigma_{v}\right\}$ if we exclude from our velocity space all the orbits that do not lead to a pericentre passage at the times $t_{1}, t_{2}$ corresponding to the two bursts of star formation at $t_{2} \sim 6 \mathrm{Gyr}$ and $t_{1} \sim 2 \mathrm{Gyr}$. Moreover, we do not pretend to know the precise moment for the time of closest approach, but we can provide the time range $\delta t_{i}, i=1,2$ (e.g.,

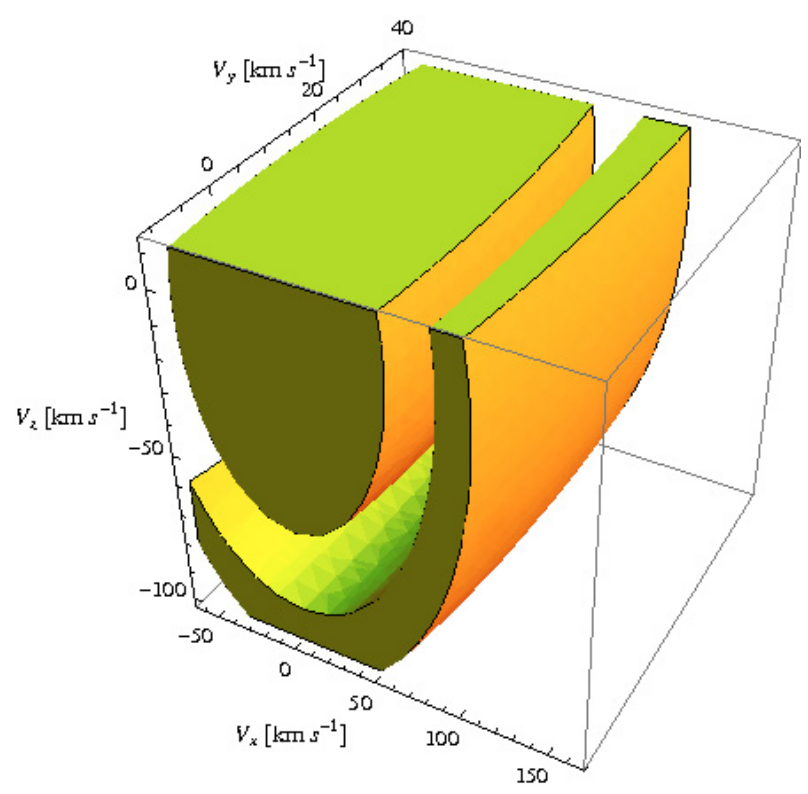

Fig. 6. $T_{x_{\mathrm{Car}}} \mathbb{Q}$ at $t=0$.

Hurley-Keller et al. 1998). In general we expect $\delta t_{2}>\delta t_{1}$ and that both are not smaller than 1 Gyr. The conditions we want to implement in our minimization tool can be achieved by requiring a minimum in the distance function $\left.\mathrm{d}\left(t, \boldsymbol{v}_{0}\right)\right|_{\mathrm{Car}}: V^{3} \times \mathbb{R} \rightarrow \mathbb{R}$ in the range $\left[t_{1}-\frac{\delta t_{1}}{2}, t_{1}+\frac{\delta t_{1}}{2}\right]$ and in the range $\left[t_{2}-\frac{\delta t_{2}}{2}, t_{2}+\frac{\delta t_{2}}{2}\right]$ :

$$
\left.\partial_{t} \mathrm{~d}\left(t, \boldsymbol{v}_{0}\right)\right|_{t=\hat{t}}=\left.0 \wedge \partial_{t, t} \mathrm{~d}\left(t, \boldsymbol{v}_{0}\right)\right|_{t=\hat{t}}>0 \text {. }
$$

Here, clearly the continuity of the partial derivative is assumed with respect to the time $\partial_{t}$. We underline immediately that, as shown by Eq. (5), this condition does not at all require excluding other pericentre passages. In the case of the manifold $T_{x_{\mathrm{Car}}} \mathbb{Q}$ at $t=0$ we show initial velocity space in Fig. 6 where we can see the section within which the condition expressed by Eq. (5) is satisfied. This condition clearly represents a good constraint when compared with the whole space where we want to find the minimum of the action. The advantage is in the more restricted number of points that we have to sample, for which we have to integrate the differential equation and to solve the action integral, as well as in the reduced computational time.

At this stage, we assume here that the tidal field acts as a trigger for the star formation. The proof of this assumption will be provided later with a self-consistent generation of the star formation history of Carina in the contest of the smooth hydrodynamic particle approach integrated in a full $N$-body simulation.

Nevertheless, by excluding those orbits that do not show any pericentre passage in the distance range required by matching Carina's SFH will reduce the space further where we have to minimize the action (and the computational time required for the minimization!). We adopt the same methods of minimization as in Pasetto \& Chiosi (2007). We point out that the method applied here does not require any previous orbit parameterization as in a cosmological contest (e.g., Peebles 1989). Moreover, the alternative approach that comes from the Fourier series decomposition used in stellar spectral dynamics (e.g., Carpintero \& Aguilar 1998) cannot be exploited here because of the small number of orbital periods during which we can integrate the dwarf galaxy (no more than 9 Gyr and an orbital period between 2 and 4 Gyr) could lead to wrong frequency determinations. Nevertheless, the full numerical integration of the action works fine for this small 
number of orbital periods. The only limitation of our method is the treatment of the mass loss in Eq. (4): an approach for a dwarf galaxy experiencing a strong dwarf-bulge interaction is not able to reproduce correctly the orbits we obtain with a full $\mathrm{N}$-body simulation. This limits our methodology to the more distant dwarfs such as the Carina dwarf galaxy.

With this approach, we can generate a family of orbits with the following values (obtained through the minimization process of the action for the Carina dwarf galaxy starting at $t_{l b}=$ 9.645 Gyr):

$\boldsymbol{x}_{\mathrm{Car}}=(-73,-64,3) \mathrm{kpc}$,

$\boldsymbol{v}_{\mathrm{Car}}=(65,-115,75) \mathrm{km} \mathrm{s}^{-1}$.

These are the initial phase-space values for most probable volume of $T_{x_{\mathrm{Car}}\left(t_{l b}=9.645 \mathrm{Gyr}\right)} \mathbb{Q}$ for the galaxy at the initial time of our $N$-body simulation.

After a full $N$-body simulation run, the previous initial conditions led to the following present-day best-fit values (values for the barycentre of the galaxy in a full $N$-body integration at $\left.t_{l b}=0\right)$ :

$\boldsymbol{x}_{\mathrm{Car}}=(22,89,-34) \mathrm{kpc}$

$\boldsymbol{v}_{\mathrm{Car}}=(-136,13,-45) \mathrm{km} \mathrm{s}^{-1}$

(values in the reference system $S_{0}$ ), which are fully compatible with the proper motion, radial velocity, and distances that are actually observed.

\subsection{Robustness of the point-mass solutions}

The robustness of the the point-mass integration is the subject of many cosmological or pure dynamical studies but often without self-consistent consideration of the star formation processes (e.g., Peñarrubia \& Benson 2005; Lux et al. 2010). The relation between star formation and orbit determination is fully exploited here for the first time as a key ingredient for constraining the Carina orbit determination. Nevertheless, once we have determined a family of solutions as in Eq. (6), and before generating a complete $N$-body integration that self-consistently considers the structural parameters of Carina and its star formation history, we check this family of orbits against the possible role that dynamical friction could have played (Chandrasekhar 1943) and the parameters of the potential.

\subsubsection{The dynamical friction}

In the last century, the perturbative methods applied to dynamical friction studies have achieved several important results in order to overcome the limitation of the original formulation in the celebrated article by Chandrasekhar (i.e. mainly the isotropy of the surrounding stellar field, see Tremaine \& Weinberg 1984; Weinberg 1986). Closely related to this approach is also the work of Bekenstein \& Maoz (1992); Nelson \& Tremaine (1999) which related the dynamical friction to the fluctuation-dissipation theorem. We follow the treatment presented in the paper of Colpi \& Pallavicini (1998), which contains the Chandrasekhar work as a special limit (e.g., Kandrup 1983). In this formulation of the perturbative approach (extension of previous works of e.g., Kandrup 1981; Seguin \& Dupraz 1994, 1996) the authors recollect and extend several important results in order to overcome the limitation of the Chandrasekhar formula that allow one to analytically follow the gravitational wake influence, the tidal deformation, and the shift of the barycentre of the primary galaxy.
Moreover, this formulation unifies the local and large-scale interpretation of dynamical friction and provides a treatment that is even superior to the full $N$-body simulations often limited by the number of particles (see Colpi et al. 1999). What is more interesting for us is that in this last paper, the authors already proved that the role of the dynamical friction for the MW dwarf satellite is marginal, especially with reference to the more distant dwarfs, and in conflict with what would be expected from the previous, straightforward application of the Chandrasekhar formula. Finally, if we want to perform a simple check under the approximation presented in the paper of Colpi et al. (1999) with the further simplification that comes from the constraint on the previously investigated relevant pericentre distance at which the orbit of Carina is expected to evolve have its pericentre distance, we can write rewrite the equation of motion in a system, say $S_{1}$, as

$\mu \frac{\mathrm{d}^{2} \boldsymbol{x}(t)}{\mathrm{d} t^{2}}=-G N m \frac{\boldsymbol{x}(t)}{\|\boldsymbol{x}(t)\|^{3}} \int_{r^{\prime}<\boldsymbol{x}(t)} \mathrm{d}^{3} \boldsymbol{x} \rho\left(r^{\prime}\right)+\boldsymbol{F}_{\Delta}$,

where $G=4.4926 \times 10^{-6} \mathrm{kpc}^{3} \mathrm{Gry}^{-2} M_{\odot}^{-1}$ is the gravitational constant, $\mu=\frac{M_{\mathrm{MW}} M_{\mathrm{Car}}}{M_{\mathrm{Car}}+M_{\mathrm{MW}}}$ the reduced mass of Carina and MW, $\rho$ the halo density profile, and $\boldsymbol{F}_{\Delta}$ the back reaction force as presented in Appendix of Colpi et al. (1999). An alternative, faster integration, which agrees its results given the distance of Carina, is based on a time-dependent Coulumb logarithm $\ln \Lambda \sim \ln \left\{\frac{r_{\text {apo }}-R(t)}{\varepsilon}\right\}$ with $\varepsilon \sim \frac{G M}{V^{2}}$ (see White 1976; Weinberg 1989) used in the the infinite, homogeneous, non-self-gravitating collisionless background formulation by Chandrasekhar:

$\boldsymbol{F}_{\Delta}=-4 \pi(G M)^{2} \rho \ln \Lambda\left(\operatorname{Erf}(x)-\frac{2 x}{\sqrt{\pi}} e^{-x^{2}}\right) \frac{\boldsymbol{V}}{\|\boldsymbol{V}\|}$,

where $x=\frac{\|\boldsymbol{V}\|}{\sqrt{2} \sigma}$ and $\boldsymbol{V}$ si the velocity vector of Carina.

The application of this formalism to our family of orbits for Carina confirms that the role of dynamical friction on our orbit determination is irrelevant as shown in Fig. 5. The orbit computed with Eq. (8) almost precisely overlaps the orbit determined without the dynamical friction (Sect. 2.4), so we plotted the bottom of the distance between the two orbits with a thin solid black line. We stress for comparison that the error in the knowledge of the present location of the Carina dwarf galaxy is of about $10 \mathrm{kpc}$ in radial distance (e.g., Mateo et al. 1998).

\subsubsection{The MW potential}

The role of the MW potential is fundamental in evaluating the orbit of Carina. Recently Law et al. (2009) has proved the necessity of fully exploiting the triaxiality of the MW potential to explain the tidal streams' location and the orbit of Sagittarius. Unfortunately the situation for Carina is much more complicated. The error in its present location and the absence of long tidal streams that can somehow track the orbits (or on the contrary can constraint the MW potential) prompted us to apply a different technique of taking the star formation history as a new constraint on the orbit; i.e., we are explicitly assuming that Carina's unusual star formation history was influenced by encounters with the MW.

As an illustrative example, we point out here how the existence of the bulge $\left(\sim 10^{10} M_{\odot}\right)$ can be neglected for the Carina orbit integration! Carina is orbiting in zones where the relative influence of the potential is mainly dominated by the halo and partially by the disk: $\Phi_{\text {bulge }}(\boldsymbol{x}, t) / \Phi_{\text {tot }}(\boldsymbol{x}, t)<0.1$ for every point 
$\boldsymbol{x}$ and instant $t$ of interest in our orbit determination. By using the best-fit orbit determination parameters (see Sect. 2.4, Eq. (6)) in Fig. 5, we plotted the orbit distance computed without the bulge with the dotted blue line. As evidence in the bottom of the plot, the distance between this orbit and the red orbit for our best solution retains a value comparable with the error bar as large as the present-day Carina distance uncertainty $(10 \mathrm{kpc})$. This result shows that the role of the bulge is marginal in the orbit of Carina.

\section{3. $\mathbf{N}$-body integration}

The search for the best initial conditions of the orbital parameters of Carina must include the matching of the internal properties of the dwarf. To accomplish this task, we performed a large number of simulations in the space of the restricted initial orbital condition, which surround the best-fit values obtained from the minimal action in the previous section.

To perform this analysis in an automatic way, we wrote a code that computes all of the desired properties (derived star formation history, chemistry, and kinematic properties of the orbiting evolved object) centred on the non-inertial barycentre of the particles representing the stellar component of the dwarf, say $S_{2}$. The barycentre of the moving dwarf's stellar component also has to be automatically computed to speed up analysis of the dwarf's orbits. This is performed with an original method based on the down hill simplex algorithm (explained in Appendix B).

A detailed description of the implementation adopted for the star formation recipes, chemical evolution, and dynamics for this GRAPE-treeSPH code can be found in Berczik (1999), Berczik et al. (2003a), Berczik et al. (2003b), Berczik et al. (2002), Berczik \& Petrov (2001), Berczik (2000) and references therein. Special purpose hardware (Spurzem et al. 2009) was used to carry out several high-resolution chemo-dynamical simulations.

\subsection{Initial conditions}

The structural properties were adopted from an extension of the isolated models presented in Pasetto et al. (2010) in order to include a larger set of scale length initial conditions (Pasetto et al. 2010, in prep.). Those isolated models then are evolved in the orbits around the MW selected as explained in the previous section. Star formation history, chemistry, and dynamical properties of the Carina dwarf galaxy are self-consistently determined during the 9 Gyr of evolution.

The initial condition can be partially tuned with the help of the literature results for Carina. Colour magnitude diagrams were studied in Hurley-Keller et al. (1998), Mighell (1997, 1992, 1990b,a); Monelli et al. (2006b,a, 2004b,a,c, 2003a,b); Rizzi et al. (2004), and Rizzi et al. (2003). Spectroscopic measurements yielded chemical abundances e.g., Koch et al. (2006, 2008), the $M / L$ ratio (e.g., Gilmore et al. 2007) and the dark matter distribution (e.g., Hayashi et al. 2003; Gilmore et al. 2007). From the analysis of the CMD of Carina (e.g., Hurley-Keller et al. 1998) we inferred that the oldest stellar population contributes no more than $15 \%$ of the stellar mass, and from the work of Mateo et al. (1993) we got a mass-to-light ratio in the $V$ band of $\left(\frac{M}{L}\right)_{V} \sim 23\left(\frac{M_{\odot}}{L_{\odot}}\right)_{V}$. Thus from Table 3 of Hayashi et al. (2003), we got $L=0.4 \times 10^{6} L_{\odot}$, which led to a stellar mass estimate of $M \approx 23 \times 0.4 \times 10^{6} M_{\odot}=9.8 \times 10^{6} M_{\odot}$. If we now assume that the mass lost by tidal interactions could reach an order of $95 \%$, following e.g. Hayashi et al. (2003) we get $M_{\text {Car }}=2 \times 10^{8} M_{\odot}$ and, with intial dark matter contents, at least 50 times the baryonic fraction Hurley-Keller et al. (1998).
Table 2. Parameters for the galaxy model evolved in orbits around the potential.

\begin{tabular}{lcc}
\hline \hline & $t_{l b}=t_{0}$ & $t_{l b}=0$ \\
\hline 1 & & $\cong 260[\mathrm{deg}]$ \\
$\mathrm{b}$ & & $\cong-22[\mathrm{deg}]$ \\
$\mathrm{d}$ & & $\cong 100[\mathrm{kpc}]$ \\
$V_{\text {l.o.s. }}$ & & $\cong 219\left[\mathrm{~km} \mathrm{~s}{ }^{-1}\right]$ \\
$M_{\text {gas }}$ & free parameter & $\cong 0$ \\
$M_{\text {star }}$ & $1.998 \times 10^{7} M_{\odot}$ & $\cong 1.9 \times 10^{6} M_{\odot}$ \\
$M_{\text {dark }}$ & $1.978 \times 10^{8} M_{\odot}$ & $0.675 \times 10^{8} M_{\odot}$ \\
\hline
\end{tabular}

The baryonic material consists of $15 \%$ stars, $85 \%$ gas. We thus adopted a starting value of $M_{\text {gas }}=\frac{M_{\text {dark }}}{50} \frac{85}{100}=3.3 \times 10^{6} M_{\odot}$ and $M_{\text {star }}=\frac{M_{\text {dark }}}{50} \frac{15}{100}=5.9 \times 10^{5} M_{\odot}$ as guess values for our inital model. These numbers can be considered as starting values for an orbiting dwarf galaxy, around which we want to find our best-fit initial values. The final best-matching initial condition is assumed as in Table 2. The models are represented by a 3-component, self-gravitating system where the family of the multi-gamma models was adopted for the density profiles (e.g., Dehnen 1993; Tremaine et al. 1994). The scale parameters for the initial models were explored around the values of $r_{c} \cong 0.72$ for the gaseous and stellar components and of $r_{c} \cong 2.2 \mathrm{kpc}$ with a cut off around $20 \mathrm{kpc}$ for the dark matter component. The initial inner slope of the density profile was chosen around $\gamma=2$ to produce a massive and cuspy dark matter profile, while a shallower profile with $\gamma=3 / 2$ was adopted for the gas and stellar profiles.

Considering the preliminary results on the orbit analysis from Sect. 2.4, models with an initial cuspy density distribution for the dark matter component have to be preferred for Carina to survive four pericentre passages. Models with a starting relevant amount of gas suffer a flattening phenomenon of the DM density profile by the star formation processes as evidence in Pasetto et al. (2010). Flatter DM density profiles have a reduced probability of surviving different pericentre passages (e.g., Peñarrubia et al. 2010).

The initial temperature for the gas can be inferred by assuming a spherical collapse model for the initial dark matter model and a matter power spectrum $P(k)$ at redshift zero that is compatible with studies from the SDSS, such as Tegmark et al. (2004) or work on the analysis of the Lyman- $\alpha$ forest (Gnedin \& Hamilton 2002). In this case the typical RMS internal velocity of a halo within $M \leq 10^{8} M_{\odot}$ is less than $13 \mathrm{~km} \mathrm{~s}^{-1}$, so we assumed an initial sound speed in hydrogen of roughly around $v_{\mathrm{s}} \cong 10 \mathrm{~km} \mathrm{~s}^{-1}$ corresponding to a temperature of $T \cong 10000^{\circ} \mathrm{K}$.

Starting with a three-component model, we implicitly assume our evolution starts after the oldest stellar population had settled to form the central nucleus of the Carina dwarf galaxy. Any burst of star formation that occurred earlier than 9-10 Gyr ago cannot help us trace the orbital parameters back. Moreover, the timescale permitting the initial protocloud to collapse requires no less than $3 \mathrm{Gyr}$ in order to form the initial old population, and finally to settle on the orbit (according to e.g., the chemical enrichment models, Marcolini et al. 2006). Thus our orbit determination will start on a possible Carina dwarf galaxy configuration of 9 Gyr ago.

\subsection{Match with the observations}

The dynamical analysis we present shows the best-fitting model for the data as compared to the observation of the surface density 

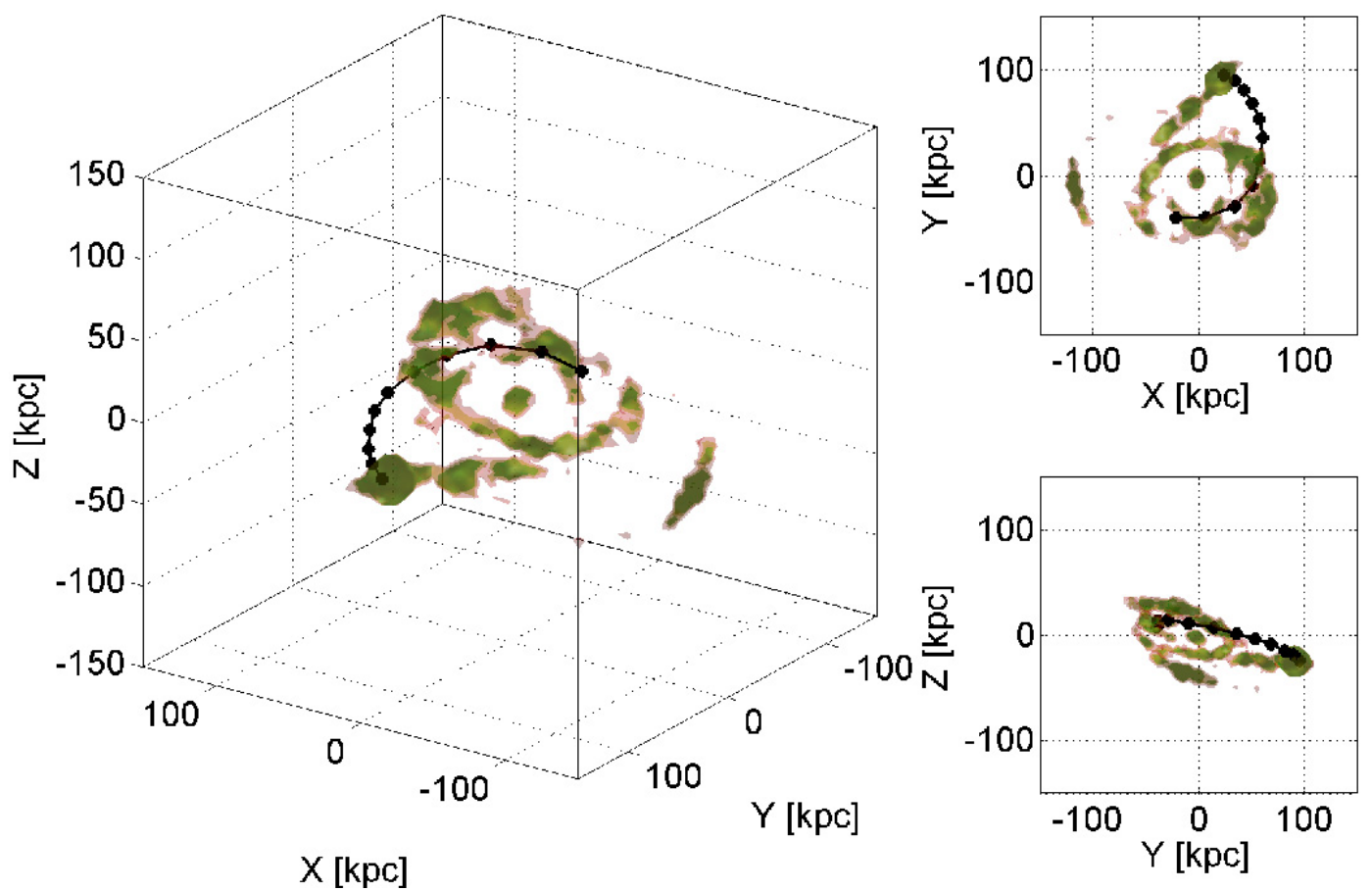

Fig. 7. $N$-body representation of the evolved galaxy. The classical point representation has been substituted with an 3D isocontour plot by counting the amount of stars within a 3D grid spanning the whole configuration space of the orbit and joining cells with equal number of stars. The green isocontours show overdensities of $1 / 10^{4}$ and the purple for overdensities of $1 / 10^{3}$.

profile of Irwin \& Hatzidimitriou (1995), and has more recently been updated in Muñoz et al. (2006) or Walker et al. (2007) with a radial velocity dispersion profile. The assumed initial and final best-fit model is presented in Table 2.

With the adopted numerical scheme the number of particles is not constant; see e.g., Pasetto et al. (2010); Berczik (1999); Berczik \& Kravchuk (2000) and references therein for detailed formulation of the star formation processes. The starting models are described with 350000 particles growing with time up to half a million particles, and the reference model we are using for the match with current observations (after 9 Gyr of evolution) contains about $1.2 \times 10^{5}$ stars within the $5 \mathrm{kpc}$ from the barycentre of the Carina dwarf galaxy.

An illustrative sketch of the evolution of the system after about 9.6 Gyr of evolution is presented in Fig. 7. The plot shows several low-resolution streams where the mass density drops down to $1 / 10^{2} \div 1 / 10^{3}$ of the total mass thus showing clearly how the tidal tails developed by the Carina dwarf galaxy are expected to be extremely diffuse after $9 \mathrm{Gyr}$ of evolution (i.e., now). In this sense, the more diffuse zones have characteristics that the model can predict but where the observations are lacking or their interpretation is fragile, e.g. Majewski et al. (2005). The last snapshots of the orbit evolution are also presented with a solid black line. We observe the general "tidal tail flipping" phenomenon already evident in high-resolution simulations by e.g., Klimentowski et al. (2009). The model at a generic snapshot is analysed in a reference system centred on the baryonic matter barycentre as seen in Fig. 8.

We use Fig. 8 to illustrate how the tidal tails developed in the simulation since the first pericentre-passage, allowing us to define an ellipsoid as soon as we plot the 3D contours for even more diffuse regions. In Fig. 8 a yellow ellipsoid is visible in the center for which we can easily compute the inertia matrix to define its principal axis. Once the principal axes along the tidal tail direction are determined, we can perform our analysis along this

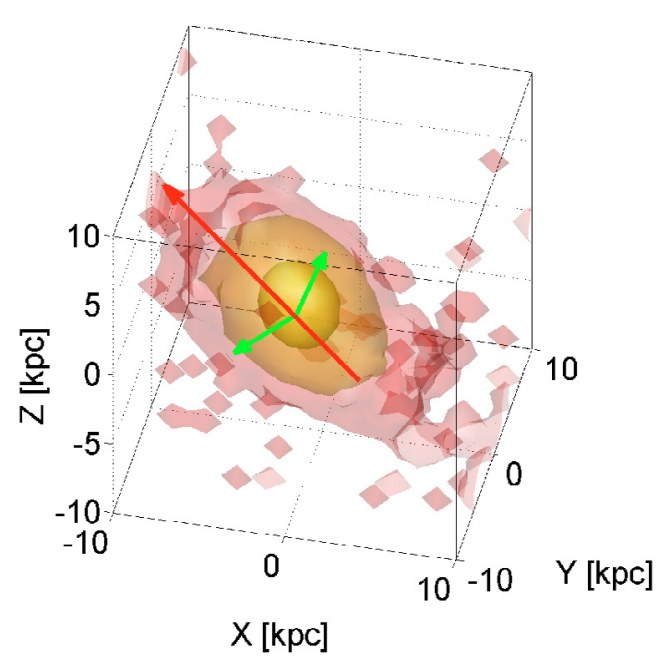

Fig. 8. A zoom of the previous figure centred on the dwarf galaxy system $S_{2}$. The red arrow shows the eigenvector direction pointing in the radial direction where the radial profiles are computed (see text for details). We have not performed our analysis in comparison with the observation along the l.o.s. thanks to the spherical symmetry developed by the system at $t=0$.

radial direction (e.g., the red arrow in this figure). However, we underline that the radial stellar profiles in the orthogonal principal directions present similar profiles not showing any pronounced ellipsoidal shape at $t=9.6 \mathrm{Gyr}$ (thus providing good agreement with the observational approach used in Muñoz et al. 2006).

\subsubsection{Surface density profile}

Figure 9 shows a plot of the density profile and the data from Muñoz et al. (2006) where we have exploited the true physical 


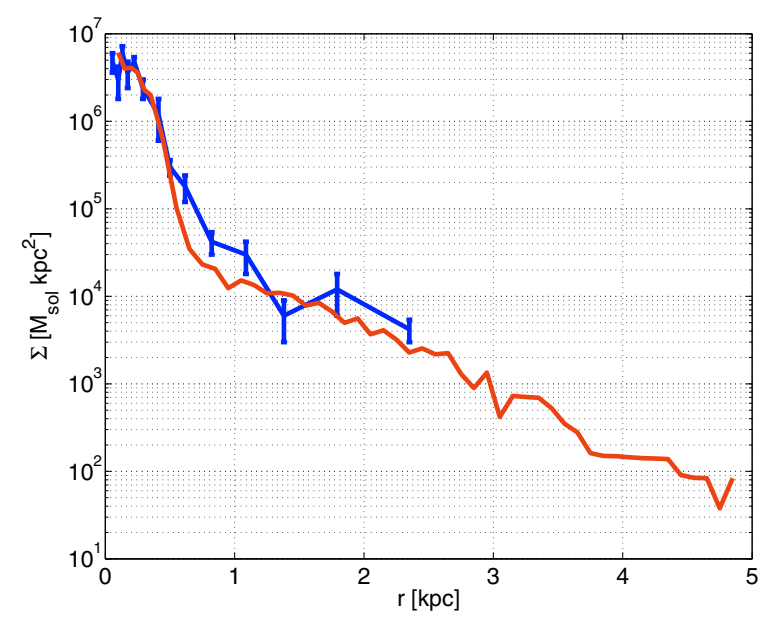

Fig. 9. Surface density from observational data and the baryonic profile deduced from the observation. The model is in red and the data with the error bars are shown in blue.

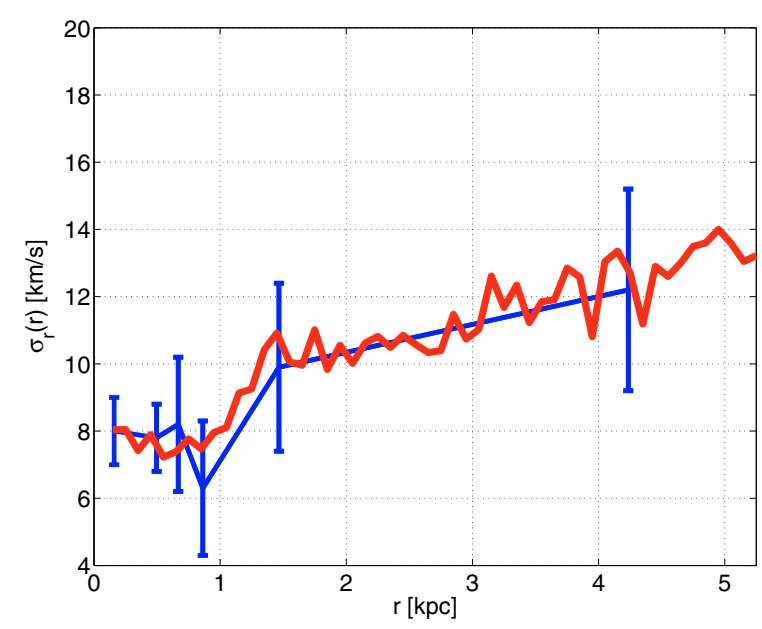

Fig. 10. Radial velocity dispersion profile as computed from the model (in red) and the observation (in blue with the error bars).

dimensions instead of the angular dimensions for the system and the data representation because they are an immediate indicator of the dynamical range we are considering; e.g., the surface on the celestial sphere can be calculated in spherical coordinates $\Sigma=d_{\operatorname{Car}} \int_{\alpha}^{\beta} \mathrm{d} \varphi \int_{\alpha}^{\gamma} \mathrm{d} \theta \sin \theta$, where $\alpha, \beta, \gamma$ are angular borders of the CCD mosaic of observational data, suitably converted from arcmin to radian.

An immediately evident result of our simulations is the reproduction the observed change in the slope of the surface density profile (e.g., Muñoz et al. 2006) which fit the observational data quite well.

\subsubsection{Radial velocity dispersion}

The other property we can now match is the radial velocity dispersion $\sigma_{r}(r)$, which is typically considered a fundamental indicator of the dynamical model of the galaxy if analysed with the Jeans equation for spherical-stationary systems (Fig. 10). The best-matching model nevertheless shows compatibility with the general trend of the baryonic matter traced by the observations. The general decline of the velocity dispersion $1.5 \mathrm{kpc}$ is also reproduced as a feature of the model. (Compare it with the change
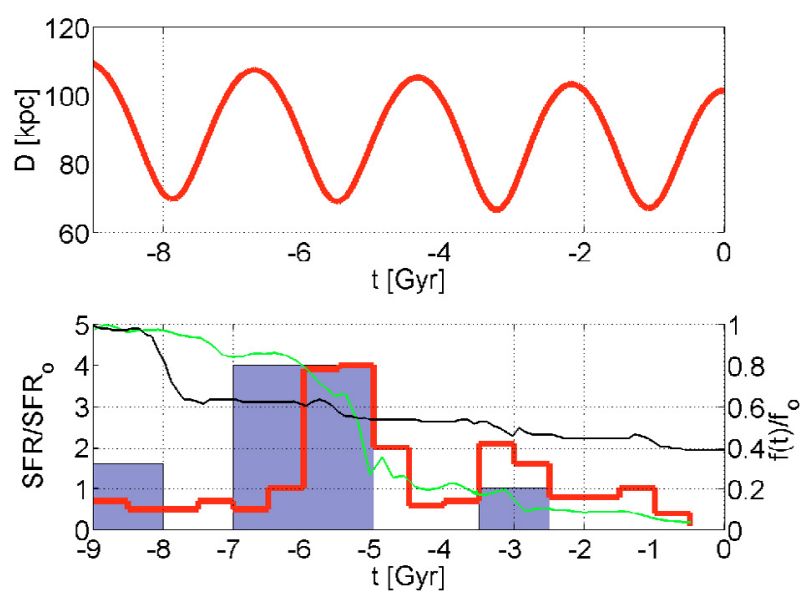

Fig. 11. Star formation rate (SFR) as a function of time normalized to the maximum of the observed SFR $\left(S F R_{o}\right)$. The upper panel shows the radial distance of the $N$-body system to the origin of the reference system centered on the MW's barycentre for the best-fitting orbit. The lower panel shows the star formation triggered by the pericentre passages and we report the profile of SFR as deduced in Rizzi et al. (2003). In the lower panel thin green and black lines refer to the gas and bound mass fraction normalized to the initial value $\left(f(t) / f_{0}\right.$ on the right vertical axis).

of the slope of the surface density profile shown in Fig. 9.) The innermost velocity dispersion value is around $8 \mathrm{~km} \mathrm{~s}^{-1}$.

\subsubsection{Star formation history}

Finally we present the star formation history obtained from the $\mathrm{N}$-body simulations. The criteria for star formation and the evolution of the isolated model were adopted from Pasetto et al. (2010). The resulting SFH for the orbiting model has been normalized here to the the highest observational peak in the star formation history deduced from the observations by Rizzi et al. (2003).

This approach is mainly due to the impossibility of adequately resolving a dwarf galaxy with a one-to-one correspondence between stars and particles. The true galaxy Carina will probably contain several million stars, and only a small fraction of them can be represented with an $N$-body simulation. As a consequence, $\frac{\mathrm{d} M}{\mathrm{~d} t}$ depends on the resolution of our dwarf galaxy model. The consequence of this limitation is that the initial amount of gas that we have to assign to the dwarf galaxy model for Carina when it starts to evolve is still a free parameter that depends on the gas consumption that led to formation of its oldest stellar population and on the gas consumption of the self-consistently triggered star formation. Our goal has been to adapt the initial amount of gas such that there is no gas left after the last burst of star formation. This should be able to reproduce a Carina dwarf at the present time where essentially no gas is observed.

While we are unable to impose strong constraints on this initial gas fraction, and to reproduce the induced burst of star formation, we present in the simulated SFH normalized to the observed SFH Fig. 11. We emphasize that we are not claiming to reproduce the true star formation history, but that we simply underline how tuned initial conditions are able to reproduce a system with present-day properties comparable to Carina including a star formation history dominated by well-separated star formation episodes. In particular, to obtain the star formation of the Carina dwarf galaxy, we have to tune 
- a suitable amount of gas that survives the initial phase of SNII in the center of Carina, and that initially coexists with the primordial oldest stellar population;

- the original location of this dwarf galaxy that needs to be located not so far from the first pericentre passage. The first tidal pericentric passage may not extinguish the internal star formation processes;

- the amount of dark matter at the initial condition that will survive the first pericentre passage stripping.

If the simulations are started prior to the apocentre passage $\left(t_{l b}<10 \mathrm{Gyr}\right)$, the first infall into the potential well of MW galaxy, which takes approximately $1 \mathrm{Gyr}$, causes too early a gas dissipation and dark matter stripping, making us unable to reproduce SFH-like Carina. For this reason, this alternative scenario of formation of the Carina dwarf galaxy has not been investigated further.

These conditions for the initial gas fraction and orbital initial conditions represent our chosen initial physical parameters to obtain the specific Carina SFH as an output that match the observations. As a general trend, we see from Fig. 11 that the star formation after the first passage at the pericentre is typically more active. The galaxy produces an evident increase in the star formation rate around $6 \mathrm{Gyr}$ ago and some smaller peaks correlated with the subsequent pericentre passages. A general trend is toward the skewness of the star formation peaks, showing a rapid rise in the early phases and slower decline after the point of closest approach to the primary. In the same figure we present the evolution of the bound mass fraction and the gas fraction. The initial amount of dark matter in the dwarf galaxy permits its baryonic component to make the first pericentre passage almost unperturbed, shielded within the dark matter potential well. The dark matter result is strongly stripped during the first pericentre passage, while the stripping results are less important in the following pericentre passages. We find that gas consumption is roughly a monotonically decreasing function of time, controlled by the star formation episodes triggered by the orbital pericentre passages (see Revaz et al. 2009, for a different approach based on completely isolated models and different SNII recipes with respect to Pasetto et al. 2010).

\section{Discussion and conclusions}

In this paper, we presented an extensive treatment of the evolution of an orbiting Carina-like dwarf galaxy, and we selfconsistently derived the star formation history. Efforts were made to consider all of the available observational constraints. Our methodology, based on the minimum action and developed to investigate all possible phase-space initial conditions, leads to a family of orbits able to reproduce using system compatible with the observational constraints full $N$-body simulation. Once the orbital parameters that minimize the action in a realistic MW potential were derived, the dynamical properties and the star formation history of Carina were recovered.

A previous study that attempted to interpret the observational constraints on Carina has recently been presented by Muñoz et al. (2008). However, in this paper the authors neglected the influence of star formation processes in their match with the observational data. Their work is based on a single component model, probably the dark matter, which is tuned to match the kinematic properties of the baryonic matter, thus directly fitting a onecomponent model to match the observed properties. Another important difference between our work and the work by Muñoz et al. (2008) is in their use of a static external potential: by increasing the size of the MW halo during the time range of evolution (and keeping all the other parameter fixed) we can vary the instants of the pericentre-passages of Carina dwarf galaxy in dependence on the initial conditions allowed by the proper motion error bars. Finally, the probability of an interaction with the Magellanic Clouds by Muñoz et al. (2006) is separately considered in Appendix C.

In our work, the star formation history seems mainly driven by tidal processes, contrary to suggestions in other recent works (e.g., Piatek et al. 2003). To quantify the influence of the environment on the star formation rate is always a difficult task not only for dwarf galaxies (e.g., Kennicutt et al. 1994), but also Lee et al. (2009) showed how, for the local environment, a bursty SFH has to be statistically preferred (see also Grebel 1997; Mateo 1998; or Rizzi et al. 2003). Nevertheless, we do not claim to have found the true reason for the episodic star formation history that Carina experienced in the past. We have just presented a possible interpretation in the framework of the dynamical interactions. The details of the interplay with the environment still need to be fully understood and reproduced for interacting dwarfs (e.g., Bouchard et al. 2009). However, by tuning the amount of gas that is collapsing in a dark-matter dominated protocloud of Carina (retained despite the initial phase of Supernovae type II explosions), a quite similar star formation history for the Carina can be reproduced self-consistently as the result of a direct reaction by the system to the MW tidal field.

Once other processes emerge as clearly influencing the amount of gas during the history of the Carina dwarf galaxy, e.g., the effects of ram-pressure stripping as a possible key ingredient to clean the bulk of interstellar matter from dwarf galaxies (Grebel et al. 2003), then the initial amount of gas has to be tuned again, but with no influence on our orbit determination. Nevertheless, the expected density for the MW gaseous halo is probably too low (e.g., Murali 2000) to strongly influence the gas evolution of the inner part of the Carina dwarf galaxy which orbits between 60 and $100 \mathrm{kpc}$ from the MW center (see, e.g., Fig. 4).

Acknowledgements. We are grateful to an anonymous referee for improving this paper. Simulations were performed partially on the GRACE supercomputer (grants I/80 041-043 of the Volkswagen Foundation and 823.219-439/30 and 136 of the Ministry of Science, Research and the Arts of Baden-Württemberg) and partially with the supercomputers at John von Neumann - Institut füer Computing (NIC) (NIC-project number 2979). P.B. acknowledges the special support by the NAS Ukraine under the Main Astronomical Observatory GRAPE/GRID computing cluster project. P.B.'s studies are also partially supported by the program Cosmomicrophysics of NAS Ukraine.

\section{References}

Abia, C., de Laverny, P., \& Wahlin, R. 2008, A\&A, 481, 161 Azzopardi, M., Lequeux, J., \& Westerlund, B. E. 1985, A\&A, 144, 388 Azzopardi, M., Lequeux, J., \& Westerlund, B. E. 1986, A\&A, 161, 232 Battaglia, G., Helmi, A., Morrison, H., et al. 2005, MNRAS, 364, 433 Bekenstein, J. D., \& Maoz, E. 1992, ApJ, 390, 79

Berczik, P. 1999, A\&A, 348, 371

Berczik, P. 2000, Kinematika i Fizika Nebesnykh Tel Supplement, 3, 91 Berczik, P., \& Kravchuk, S. G. 2000, Astron. Astrophys. Trans., 18, 829 Berczik, P. P., \& Petrov, M. I. 2001, Kinematika i Fizika Nebesnykh Tel, 17, 213 Berczik, P., Hensler, G., Theis, C., \& Spurzem, R. 2002, Ap\&SS, 281, 297 Berczik, P., Hensler, G., Theis, C., \& Spurzem, R. 2003a, Ap\&SS, 284, 865 Berczik, P., Hensler, G., Theis, C., \& Spurzem, R. 2003b, Commmunications of the Konkoly Observatory Hungary, 103, 155

Besla, G., Kallivayalil, N., Hernquist, L., et al. 2007, ApJ, 668, 949

Binney, J., \& Merrifield, M. 1998, Galactic astronomy (Princeton series in astrophysics) 
Bouchard, A., Da Costa, G. S., \& Jerjen, H. 2009, AJ, 137, 3038 Bovy, J., Hogg, D. W., \& Rix, H. 2009, ApJ, 704, 1704 Brown, W. R., Geller, M. J., Kenyon, S. J., \& Diaferio, A. 2010, AJ, 139, 59 Burkert, A. 1997, ApJ, 474, L99

Cannon, R. D., Hawarden, T. G., \& Tritton, S. B. 1977, MNRAS, 180, 81P Carpintero, D. D., \& Aguilar, L. A. 1998, MNRAS, 298, 1

Carraro, G., Lia, C., \& Chiosi, C. 1998, MNRAS, 297, 1021 Chandrasekhar, S. 1943, ApJ, 97, 255

Ciotti, L., \& Morganti, L. 2009, MNRAS, 393, 179

Cole, N., Newberg, H. J., Magdon-Ismail, M., et al. 2008, ApJ, 683, 750 Colpi, M., \& Pallavicini, A. 1998, ApJ, 502, 150

Colpi, M., Mayer, L., \& Governato, F. 1999, ApJ, 525, 720

Combes, F. 1999, Ap\&SS, 265, 417

Cook, K., Schechter, P., \& Aaronson, M. 1983, BAAS, 15, 907

Dehnen, W. 1993, MNRAS, 265, 250

Dehnen, W., \& Binney, J. 1998, MNRAS, 294, 429

Dehnen, W., McLaughlin, D. E., \& Sachania, J. 2006, MNRAS, 369, 1688

Eskridge, P. B. 1988a, AJ, 96, 1352

Eskridge, P. B. 1988b, AJ, 95, 1706

Eyre, A., \& Binney, J. 2009, MNRAS, 400, 548

Flynn, C., Holmberg, J., Portinari, L., Fuchs, B., \& Jahreiß, H. 2006, MNRAS, 372,1149

Freeman, K., \& Bland-Hawthorn, J. 2002, ARA\&A, 40, 487

Gilmore, G., Wilkinson, M. I., Wyse, R. F. G., et al. 2007, ApJ, 663, 948

Gnedin, N. Y., \& Hamilton, A. J. S. 2002, MNRAS, 334, 107

Gnedin, O. Y., Brown, W. R., Geller, M. J., \& Kenyon, S. J. 2010, ApJ, 720, L108

Gómez-Flechoso, M. A., Fux, R., \& Martinet, L. 1999, A\&A, 347, 77

Gómez-Flechoso, M. Á., \& Martínez-Delgado, D. 2003, ApJ, 586, L123

Grebel, E. K. 1997, Rev. Modern Astron. 10, 29

Grebel, E. K. 1999, in The Stellar Content of Local Group Galaxies, ed. P. Whitelock, \& R. Cannon, IAU Symp., 192, 17

Grebel, E. K., Gallagher, III, J. S., \& Harbeck, D. 2003, AJ, 125, 1926

Green, R. M. 1985, Spherical astronomy (Cambridge and New York: Cambridge University Press)

Guo, Q., \& White, S. D. M. 2008, MNRAS, 384, 2

Hayashi, E., Navarro, J. F., Taylor, J. E., Stadel, J., \& Quinn, T. 2003, ApJ, 584, 541

Helmi, A. 2004, ApJ, 610, L97

Helmi, A. 2008, A\&ARv, 15, 145

Hurley-Keller, D., Mateo, M., \& Nemec, J. 1998, AJ, 115, 1840

Irwin, M., \& Hatzidimitriou, D. 1995, MNRAS, 277, 1354

Johnson, D. R. H., \& Soderblom, D. R. 1987, AJ, 93, 864

Johnston, K. V., Zhao, H., Spergel, D. N., \& Hernquist, L. 1999, ApJ, 512, L109

Jurić, M., Ivezić, Ž., Brooks, A., et al. 2008, ApJ, 673, 864

Kallivayalil, N., van der Marel, R. P., \& Alcock, C. 2006a, ApJ, 652, 1213

Kallivayalil, N., van der Marel, R. P., Alcock, C., et al. 2006b, ApJ, 638, 772

Kallivayalil, N., van der Marel, R. P., Anderson, J., Besla, G., \& Alcock, C. 2009, in IAU Symp. 256, ed. J. T. van Loon, \& J. M. Oliveira, 93

Kandrup, H. 1981, ApJ, 244, 316

Kandrup, H. E. 1983, Ap\&SS, 97, 435

Kazantzidis, S., Mayer, L., Mastropietro, C., et al. 2004, ApJ, 608, 663

Kazantzidis, S., Abadi, M. G., \& Navarro, J. F. 2010, ApJ, 720, L62

Kennicutt, Jr., R. C., Tamblyn, P., \& Congdon, C. E. 1994, ApJ, 435, 22

Klessen, R. S., \& Zhao, H. 2002, ApJ, 566, 838

Klessen, R. S., Grebel, E. K., \& Harbeck, D. 2003, ApJ, 589, 798

Kleyna, J., Geller, M., Kenyon, S., \& Kurtz, M. 1999, AJ, 117, 1275

Kleyna, J., Wilkinson, M. I., Evans, N. W., Gilmore, G., \& Frayn, C. 2002, MNRAS, 330, 792

Klimentowski, J., Łokas, E. L., Kazantzidis, S., et al. 2009, MNRAS, 400, 2162 Koch, A., Grebel, E. K., Wyse, R. F. G., et al. 2006, AJ, 131, 895

Koch, A., Grebel, E. K., McWilliam, A., et al. 2007a, Astron. Nachr., 328, 652

Koch, A., Kleyna, J. T., Wilkinson, M. I., et al. 2007b, AJ, 134, 566

Koch, A., Wilkinson, M. I., Kleyna, J. T., et al. 2007c, ApJ, 657, 241

Koch, A., Grebel, E. K., Gilmore, G. F., et al. 2008, AJ, 135, 1580

Kochanek, C. S. 1996, ApJ, 457, 228

Koposov, S. E., Rix, H., \& Hogg, D. W. 2010, ApJ, 712, 260

Kroupa, P. 1997, New Astron., 2, 139

Kuhn, J. R., Smith, H. A., \& Hawley, S. L. 1996, ApJ, 469, L93

Lanfranchi, G. A., Matteucci, F., \& Cescutti, G. 2006, A\&A, 453, 67

Law, D. R., Majewski, S. R., \& Johnston, K. V. 2009, ApJ, 703, L67

Lee, J. C., Kennicutt, R. C., José G., et al. 2009, ApJ, 692, 1305

Lin, D. N. C., Jones, B. F., \& Klemola, A. R. 1995, ApJ, 439, 652

Lux, H., Read, J. I., \& Lake, G. 2010, MNRAS, submitted, [arXiv: 1001.1731]

Majewski, S. R., Ostheimer, J. C., Patterson, R. J., et al. 2000, AJ, 119, 760

Majewski, S. R., Skrutskie, M. F., Weinberg, M. D., \& Ostheimer, J. C. 2003, ApJ, 599, 1082

Majewski, S. R., Frinchaboy, P. M., Kunkel, W. E., et al. 2005, AJ, 130, 2677
Marcolini, A., D’Ercole, A., Brighenti, F., \& Recchi, S. 2006, MNRAS, 371, 643 Martínez-Delgado, D., Alonso-García, J., Aparicio, A., \& Gómez-Flechoso, M. A. 2001, ApJ, 549, L63

Martínez-Delgado, D., Peñarrubia, J., Jurić, M., Alfaro, E. J., \& Ivezić, Z. 2007, ApJ, 660, 1264

Mateo, M. L. 1998, ARA\&A, 36, 435

Mateo, M., Hurley-Keller, D., \& Nemec, J. 1998, AJ, 115, 1856

Mateo, M., Olszewski, E. W., Pryor, C., Welch, D. L., \& Fischer, P. 1993, AJ, 105,510

Mayer, L., Moore, B., Quinn, T., Governato, F., \& Stadel, J. 2002, MNRAS, 336, 119

McNamara, D. H. 1995, AJ, 109, 1751

Metz, M., Kroupa, P., \& Libeskind, N. I. 2008, ApJ, 680, 287

Mighell, K. J. 1990a, A\&AS, 82, 1

Mighell, K. J. 1990b, A\&AS, 82, 207

Mighell, K. J. 1992, Proc. Astron. Soc. Austr., 10, 83

Mighell, K. J. 1997, AJ, 114, 1458

Miyamoto, M., \& Nagai, R. 1975, PASJ, 27, 533

Monelli, M., Pulone, L., Corsi, C. E., et al. 2003a, AJ, 126, 218

Monelli, M., Walker, A. R., Bono, G., et al. 2003b, Mem. Soc. Astron. Ital., 74, 909

Monelli, M., Walker, A. R., Bono, G., et al. 2004a, Mem. Soc. Astron. Ital. Suppl., 5, 65

Monelli, M., Walker, A. R., Bono, G., et al. 2004b, in IAU Colloq. 193: Variable Stars in the Local Group, ed. D. W. Kurtz, \& K. R. Pollard, ASP Conf. Ser., 310,133

Monelli, M., Walker, A. W., Bono, G., et al. 2004c, Mem. Soc. Astron. Ital., 75, 114

Monelli, M., Bono, G., Nonino, M., et al. 2006a, Photometry and Spectroscopy of Bright Stars in the Carina dSph Galaxy, ed. S. Randich, \& L. Pasquini, 272

Monelli, M., Nonino, M., Bono, G., et al. 2006b, Mem. Soc. Astron. Ital., 77, 270

Mould, J., \& Aaronson, M. 1983, ApJ, 273, 530

Mould, J. R., Bothun, G. D., Hall, P. J., Staveley-Smith, L., \& Wright, A. E. 1990, ApJ, 362, L55

Muñoz, R. R., Frinchaboy, P. M., Majewski, S. R., et al. 2005, ApJ, 631, L137

Muñoz, R. R., Majewski, S. R., Zaggia, S., et al. 2006, ApJ, 649, 201

Muñoz, R. R., Majewski, S. R., \& Johnston, K. V. 2008, ApJ, 679, 346

Murali, C. 2000, ApJ, 529, L81

Nelson, R. W., \& Tremaine, S. 1999, MNRAS, 306, 1

Odenkirchen, M., Grebel, E. K., Harbeck, D., et al. 2001, AJ, 122, 2538

Oh, K. S., Lin, D. N. C., \& Aarseth, S. J. 1995, ApJ, 442, 142

Olsen, K. A. G., \& Massey, P. 2007, ApJ, 656, L61

Palma, C., Majewski, S. R., Siegel, M. H., et al. 2003, AJ, 125, 1352

Pasetto, S., \& Chiosi, C. 2007, A\&A, 463, 427

Pasetto, S., Chiosi, C., \& Carraro, G. 2003, A\&A, 405, 931

Pasetto, S., Grebel, E. K., Berczik, P., Spurzem, R., \& Dehnen, W. 2010, A\&A, 514, A47

Peñarrubia, J., \& Benson, A. J. 2005, MNRAS, 364, 977

Peñarrubia, J., Navarro, J. F., \& McConnachie, A. W. 2008, ApJ, 673, 226

Peñarrubia, J., Navarro, J. F., McConnachie, A. W., \& Martin, N. F. 2009, ApJ, 698, 222

Peñarrubia, J., Benson, A. J., Walker, M. G., et al. 2010, MNRAS, 406, 1290

Peebles, P. J. E. 1989, ApJ, 344, L53

Piatek, S., \& Pryor, C. 1995, AJ, 109, 1071

Piatek, S., Pryor, C., Olszewski, E. W., et al. 2003, AJ, 126, 2346

Piatek, S., Pryor, C., \& Olszewski, E. W. 2008, AJ, 135, 1024

Press, W. H., Flannery, B. P., \& Teukolsky, S. A. 1986, Numerical recipes. The art of scientific computing (Cambridge: University Press)

Read, J. I., Wilkinson, M. I., Evans, N. W., Gilmore, G., \& Kleyna, J. T. 2006, MNRAS, 367, 387

Reid, M. J., Menten, K. M., Zheng, X. W., et al. 2009, ApJ, 700, 137

Revaz, Y., Jablonka, P., Sawala, T., et al. 2009, A\&A, 501, 189

Rizzi, L., Held, E. V., Bertelli, G., \& Saviane, I. 2003, ApJ, 589, L85

Rizzi, L., Held, E. V., Bertelli, G., \& Saviane, I. 2004, Mem. Soc. Astron. Ital., 75,110

Robin, A. C., Reylé, C., Derrière, S., \& Picaud, S. 2003, A\&A, 409, 523

Saha, A., Monet, D. G., \& Seitzer, P. 1986, AJ, 92, 302

Sakamoto, T., Chiba, M., \& Beers, T. C. 2003, A\&A, 397, 899

Seguin, P., \& Dupraz, C. 1994, A\&A, 290, 709

Seguin, P., \& Dupraz, C. 1996, A\&A, 310, 757

Sirko, E., Goodman, J., Knapp, G. R., et al. 2004a, AJ, 127, 899

Sirko, E., Goodman, J., Knapp, G. R., et al. 2004b, AJ, 127, 914

Smecker-Hane, T. A., Stetson, P. B., Hesser, J. E., \& Lehnert, M. D. 1994, AJ, 108, 507

Smith, H. A., Kuhn, J. R., \& Hawley, S. L. 1997, in Proper Motions and Galactic Astronomy, ed. R. M. Humphreys, ASP Conf. Ser., 127, 163

Sohn, S. T., Majewski, S. R., Muñoz, R. R., et al. 2007, ApJ, 663, 960 
Spurzem, R., Berczik, P., Marcus, G., et al. 2009, Comput. Sci., 23, 231 Stoehr, F., White, S. D. M., Tormen, G., \& Springel, V. 2002, MNRAS, 335, L84

Strigari, L. E., Bullock, J. S., Kaplinghat, M., et al. 2008, Nature, 454, 1096

Tegmark, M., Blanton, M. R., \& Strauss, M. A. 2004, ApJ, 606, 702

Tolstoy, E., Irwin, M. J., Helmi, A., et al. 2004, ApJ, 617, L119

Tremaine, S., \& Weinberg, M. D. 1984, MNRAS, 209, 729

Tremaine, S., Richstone, D. O., Byun, Y.-I., et al. 1994, AJ, 107, 634

Vallenari, A., Pasetto, S., Bertelli, G., et al. 2004, in Milky Way Surveys:

The Structure and Evolution of our Galaxy, ed. D. Clemens, R. Shah, \&

T. Brainerd, ASP Conf. Ser., 317, 203

van den Bergh, S. 1999, A\&ARv, 9, 273

Walcher, C. J., Fried, J. W., Burkert, A., \& Klessen, R. S. 2003, A\&A, 406, 847
Walker, M. G., Mateo, M., Olszewski, E. W., et al. 2006a, AJ, 131, 2114

Walker, M. G., Mateo, M., Olszewski, E. W., et al. 2006b, ApJ, 642, L41

Walker, M. G., Mateo, M., Olszewski, E. W., et al. 2007, ApJ, 667, L53

Walker, M. G., Mateo, M., \& Olszewski, E. W. 2008, ApJ, 688, L75

Walker, M. G., Mateo, M., \& Olszewski, E. W. 2009, AJ, 137, 3100

Watkins, L. L., Evans, N. W., \& An, J. H. 2010, MNRAS, 406, 264

Weinberg, M. D. 1986, ApJ, 300, 93

Weinberg, M. D. 1989, MNRAS, 239, 549

Westfall, K. B., Majewski, S. R., Ostheimer, J. C., et al. 2006, AJ, 131, 375

White, S. D. M. 1976, MNRAS, 174, 467

Wilkinson, M. I., Kleyna, J. T., Evans, N. W., et al. 2004, ApJ, 611, L21

Xue, X. X., Rix, H. W., Zhao, G., et al. 2008, ApJ, 684, 1143

Zhao, H. 1998, ApJ, 500, L149 


\section{Appendix A: Determination of spatial velocities}

We update the transformation matrix $\mathbf{T}$ defined in Johnson \& Soderblom (1987) (following Green (1985)) to equinox J2000:

$\mathbf{T}=\left(\begin{array}{rrr}-0.0534 & -0.8750 & -0.4810 \\ +0.4939 & -0.4418 & +0.7488 \\ -0.8678 & -0.1975 & +0.4558\end{array}\right)$

The matrix B of Johnson \& Soderblom (1987) remains defined as:

$\mathbf{B} \equiv \mathbf{T}\left(\begin{array}{ccc}\cos (\alpha) \cos (\delta) & -\sin (\alpha) & -\cos (\alpha) \sin (\delta) \\ \sin (\alpha) \cos (\delta) & \cos (\alpha) & -\sin (\alpha) \sin (\delta) \\ \sin (\delta) & 0 & \cos (\delta)\end{array}\right)$,

where $(\alpha, \delta)$ are the equatorial coordinates of the generic dwarf galaxy at the present time $t=t_{0}$. In our case, we get $(\alpha, \delta)_{\mathrm{Car}}=$ $(1.76,-0.89) \mathrm{rad}$ for the Carina dwarf galaxy. Now we have to consider a reflection of the velocity axis that we want pointing away from the Galactic centre and obtain the correction for the rotation of the galaxy, as well as the motion of the sun relative to the local standard of rest:

$$
\left(\begin{array}{l}
v_{x} \\
v_{y} \\
v_{z}
\end{array}\right)=\left(\left(\begin{array}{ccc}
-1 & 0 & 0 \\
0 & 1 & 0 \\
0 & 0 & 1
\end{array}\right)\left(\left(\mathbf{B}\left(\begin{array}{c}
v_{r} \\
k \mu_{\alpha} d \\
k \mu_{\delta} d
\end{array}\right)\right)+\left(\begin{array}{c}
v_{x \odot, \mathrm{LSR}} \\
v_{y \odot, \mathrm{LSR}} \\
v_{z \odot, \mathrm{LSR}}
\end{array}\right)\right)\right)+\left(\begin{array}{c}
0 \\
V_{c} \\
0
\end{array}\right)
$$

where $\boldsymbol{v}=\left\{v_{x}, v_{y}, v_{z}\right\}$ is the velocity of the dwarf galaxy at the instant $t=t_{0}, v_{r}$ is the radial velocity, $\left(\mu_{\alpha}, \mu_{\delta}\right)$ the proper motions in $\operatorname{arcsec} \mathrm{yr}^{-1}, d$ the distances that have to be assumed in $\mathrm{pc}$ to apply the conversion values $k=4.74 ; \boldsymbol{v}_{\odot, \mathrm{LSR}}=$ $\{10.0,5.2,7.2\} \mathrm{km} \mathrm{s}^{-1}$ as in Binney \& Merrifield (1998) and $V_{c}=220 \mathrm{~km} \mathrm{~s}^{-1}$ as adopted by the IAU (1986). For the distances, we also adopt a different reference system from Johnson \& Soderblom (1987) in order to obtain a right-handed reference system. This is achieved by imposing a double reflection for the positive $X$-axis originating in the Galactic centre and the positive $Y$-axis pointing along decreasing Galactic longitude:

$$
\left(\begin{array}{l}
x \\
y \\
z
\end{array}\right)=\left(\begin{array}{ccc}
-1 & 0 & 0 \\
0 & -1 & 0 \\
0 & 0 & 1
\end{array}\right)\left(d\left(\begin{array}{c}
\cos (b) \cos (l) \\
\cos (b) \sin (l) \\
\sin (b)
\end{array}\right)\right)+\left(\begin{array}{l}
x_{\odot} \\
y_{\odot} \\
z_{\odot}
\end{array}\right),
$$

where $\boldsymbol{x}=(x, y, z)$ is the generic position of the dwarf galaxy, $\boldsymbol{x}_{\odot}=\left\{x_{\odot}, y_{\odot}, z_{\odot}\right\} \cong\{8.5,0.0,0.0\}$ is the Sun's position assumed for simplicity to lie in the plane of the MW and $(l, b)$ are the Galactic coordinates that can be consistently derived for a cross check from

$$
\left(\begin{array}{c}
\cos (b) \cos (l) \\
\cos (b) \sin (l) \\
\sin (b)
\end{array}\right)=\mathbf{T}^{-1}\left(\begin{array}{c}
\cos (\delta) \cos (\alpha) \\
\cos (\delta) \sin (\alpha) \\
\sin (\delta)
\end{array}\right) .
$$

The selection of an aligned reference system between the configuration space and the velocity space will permit us to treat, in a simpler form, the velocity vector as a derivative of the position vector for times different from the present. Similarly, we can derive the errors as

$$
\left(\begin{array}{c}
\sigma_{x}^{2} \\
\sigma_{y}^{2} \\
\sigma_{z}^{2}
\end{array}\right)=2 \mu_{\alpha} \mu_{\delta} \frac{\sigma_{p}^{2}}{p^{4}}\left(\begin{array}{l}
B_{12} B_{13} \\
B_{22} B_{23} \\
B_{32} B_{33}
\end{array}\right) k^{2}+\mathbf{B}^{2}\left(\begin{array}{c}
\sigma_{v_{r}}^{2} \\
\left(\frac{k}{p}\right)^{2}\left(\left(\frac{\mu_{\alpha} \sigma_{p}}{p}\right)^{2}+\sigma_{\mu_{\alpha}}^{2}\right) \\
\left(\frac{k}{p}\right)^{2}\left(\left(\frac{\mu_{\delta} \sigma_{p}}{p}\right)^{2}+\sigma_{\mu_{\delta}}^{2}\right.
\end{array}\right)
$$

where $p$ is the parallax and $\mathbf{B}^{2}$ the matrix with elements $\left(B_{i j}\right)^{2}$ $\forall i, j$. With these equations and the proper motion and errors in the text, we can derive our estimate value for the orbital energy of the dwarf galaxy we are analysing.

\section{Appendix B: Barycentre determination}

Once we try to move from the crude point mass determination to the full $N$-body description and include many different astrophysical aspects, we encounter the necessity of analysing the system properties of the many $N$-body system realizations by detecting the position of the centre of mass of the system. The determination of the not inertial centre of mass of a galaxy moving within an external force field can be performed in different ways but it is clear that we must evaluate it automatically to speed up analysis of the large number of simulations we performed for finding the best match with the observational constraints. In this Appendix, we present an original approach based on the downhill simplex method (Nelder \& Mead 1965), which is a direct search method that works moderately well in low-dimensional stochastic problems. Our task is to apply the method to find the barycentre of the nucleus of our dwarf galaxy, limiting ourselves to the knowledge of the position of the galaxy at a given snapshot $\boldsymbol{x}_{i}(t) \in \mathbb{R}^{3}$ for every particle, $\forall i$, and at every moment, $\forall t$, during the evolution of our dwarf. We refer the reader to books such as Numerical Recipes by Press et al. (1986) which uses the method in the section dedicated to the minimization already back in 1986. Here we limit ourselves to showing a further possible application where this method is suitable. In the practical implementation, we refer to the matrix formalism developed in the original work of Nelder and Mead. The simplex, a convex hull of a tetrahedron for our 3D space surrounding our MW galaxy, represented by a $3 \times(3+1)$ time-dependent matrix, whose columns are the vertex

$\alpha(\hat{t})=\left(B(\hat{t}) \boldsymbol{x}_{4}(\hat{t})\right)$,

where $B \equiv\left(\boldsymbol{x}_{1}(\hat{t}), \boldsymbol{x}_{2}(\hat{t}), \boldsymbol{x}_{3}(\hat{t})\right)$. For any simplex $\alpha \in \mathbb{R}^{3}$ we can define the matrix, $M=M(\hat{t})$, as the $3 \times 3$ matrix whose columns represent the edges of $\alpha(\hat{t})$ :

$M(\hat{t})=\left.\left(\boldsymbol{x}_{1}-\boldsymbol{x}_{4}, \boldsymbol{x}_{2}-\boldsymbol{x}_{4}, \boldsymbol{x}_{3}-\boldsymbol{x}_{4}\right)\right|_{t=\hat{t}}=B(\hat{t})-\boldsymbol{x}_{4} \hat{\boldsymbol{e}}^{T}$

and $\hat{\boldsymbol{e}}=(1,1,1)^{T}$. In this way we can, as a first step, check the degenerate character of the simplex by ensuring that the 3D volume of $\alpha(\hat{t}), \operatorname{vol}(\alpha(\hat{t}))=\frac{1}{6}|\operatorname{det}(M(\hat{t}))|>0$. As a consequence, in a Euclidean geometry the reflection, expansion, inside/outside-contraction and shrinkage computed by the algorithm will always produce a non-degenerate tetrahedron. We then define the diameter of the simplex as $\emptyset(\alpha)=\max _{i \neq j}\left\|\boldsymbol{x}_{i}-\boldsymbol{x}_{j}\right\|$ $\forall t$ where $\|$.$\| is the standard norm. Finally we define a suitable$ function for finding the best point representing a barycentre of the dwarf galaxy. If we call $d_{i}=\left\|\boldsymbol{x}^{[i]}-\boldsymbol{x}_{b}\right\|$ the distance of the $i$ th-star from the guess value of the barycentre $\boldsymbol{x}_{b}$ at the instant $t=\hat{t}$ under consideration, then we need to maximize the function

$$
f(\boldsymbol{x})=\sum_{d_{i}<r_{*}} m_{i}
$$

where $r_{*}$ is a characteristic radius of the system we want to analyse. Our experience shows that it does not have to be physically related to the system, but a suitable choice of a few kpc can definitely improve the convergence velocity and the stability of the barycentre value if related to the convergence criteria. If $f$ is a bounded function then for every non-degenerate case it can be proved for the downhill simplex algorithm (we indicate with $k$ the iteration of the algorithm) that

- the sequence $\left\{f\left(\boldsymbol{x}_{1}^{(k)}\right)\right\}$ always converges; 
- at every non-shrinking iterations $k, f\left(\boldsymbol{x}_{i}^{(k+1)}\right) \leqslant f\left(\boldsymbol{x}_{i}^{(k)}\right)$ for $1 \leqslant i \leqslant n+1$, with strict inequality for at least one variable of I;

- if there is only a finite number of shrink iterations, then

- each sequence $\left\{f\left(\boldsymbol{x}_{i}^{(k)}\right)\right\}$ converges as $k \rightarrow \infty$ for $1 \leqslant i \leqslant$ $n+1$

- if we call $f\left(\boldsymbol{x}_{i}^{(\infty)}\right)=\lim _{k \rightarrow \infty} f\left(\boldsymbol{x}_{i}^{(k)}\right)$ then $f\left(\boldsymbol{x}_{i}^{(\infty)}\right) \leqslant$ $f\left(x_{i}^{(k)}\right) \forall k$ and $1 \leqslant i \leqslant n+1$;

$-f\left(\boldsymbol{x}_{1}^{(\infty)}\right) \leqslant f\left(\boldsymbol{x}_{2}^{(\infty)}\right) \leqslant \ldots \leqslant f\left(\boldsymbol{x}_{n+1}^{(\infty)}\right)$.

- if there is only a finite number of non-shrink iterations, then all simplex vertexes converge to a single point.

All this ensures that the down hill simplex method applied to the function (B.1) will always converge if the number of shrink iteration is small (which is in our experience). We adopted a variable diameter as indicated by the subset of the stars for which we computed the barycentre, $\emptyset(\alpha)<r_{*}: \lim _{j \rightarrow \infty} r_{*}^{[j]}(\hat{t})=0$, e.g. with a physical radius converging to zero as the number of successive times that the down hill simplex method is applied, $j$, increases the assigned $\forall t=\hat{t}$. In practice, we of course chose $r_{*}$ to be slightly higher than the softening length of the gravitational potential $r_{*}>\varepsilon_{\text {star. }}$.

\section{Appendix C: Encounter with the Magellanic Clouds}

In this Appendix we discuss the probability for a fly by interaction with the Magellanic Clouds to investigate the possibility suggested by Muñoz et al. (2006). We relegate these arguments to this Appendix because they simply concern probability considerations that are derived from dynamics. The suggestion presented in the paper of Muñoz et al. (2006) comes from the analysis of only 15 stars in the local universe, too small to justify a further set of full simulations. In particular, we are interested in the recent determination of surprisingly large proper motion for the Large Magellanic Cloud (LMC) and the Small Magellanic
Cloud (SMC) (Kallivayalil et al. 2006b,a), which caused interest due to the possible implications for their evolutionary history (e.g., Kallivayalil et al. 2009; Piatek et al. 2008; Besla et al. 2007; Olsen \& Massey 2007). As explained in Kallivayalil et al. (2009), the most relevant problem faced by the integration of the orbits of the Magellanic Clouds with an expected apocentre so far from the MW barycentre, is the unknown mass distribution of the MW for distances larger than 100-200 kpc (e.g., Besla et al. 2007). With our MW galactic potential model presented in Appendix B, we cannot extend the integration of the LMC orbit much further as required to overlap the entire time with the orbit integration spanned by the minimization of the action for Carina. Thus we limit ourselves to the last $3 \mathrm{Gyr}$ of evolution in look-back time because in the potential of MW, the LMC after 3 Gyr of look-back time integration will already be more distant than $200 \mathrm{kpc}$ from the MW barycentre, thereby falsifying any phase-space derivation. Thus we accept the conclusion already presented in Besla et al. (2007) in favour of a single/first pericentre passage for the MC not more than a few hundred million years ago, and we proceed by minimizing the distance function $d_{\mathrm{Car}-\mathrm{MC}}: \mathbb{R}^{7} \rightarrow \mathbb{R}^{+}$

$d_{\mathrm{Car}-\mathrm{MC}}=d_{\mathrm{Car}-\mathrm{MC}}\left(\boldsymbol{v}_{0, \mathrm{Car}}, \boldsymbol{v}_{0, \mathrm{MC}}, t\right)$

in the 7-dimensional space of the initial values for $\boldsymbol{v}_{0, \text { Car }} \in$ $\left[\boldsymbol{v}_{0, \mathrm{Car}} \pm \delta \boldsymbol{v}_{0, \mathrm{Car}}\right], \boldsymbol{v}_{0, \mathrm{MC}} \in\left[\boldsymbol{v}_{0, \mathrm{MC}} \pm \delta \boldsymbol{v}_{0, \mathrm{MC}}\right]$ computed as in Appendix $\mathrm{A}$ and $t \in]-3,0]$ Gyr. We integrated the equation of motion here for the Magellanic Clouds, taking into account an extra term due to the dynamical friction as ascribed in Eq. (8) caused by the dependence of the force on the square of the mass of the Magellanic Clouds (e.g. $m_{\mathrm{LMC}} \cong 2 . \times 10^{9} M_{\odot}$ ) that makes the dynamical friction much more relevant than for Carina. The result is that the closest distance approach permitted between the two galaxies in the range of the possible phase-space observational errors is more than $50 \mathrm{kpc}$, completely ruling out any possible interaction within the currently suggested phase-space error range deduced from the observations. The same consideration holds for any interaction with the SMC. 